\title{
Renormalization of Chiral Nuclear Forces with Multiple Subtractions in Peripheral Channels
}

\author{
E. F. Batista, ${ }^{1}$ S. Szpigel, ${ }^{2}$ and V. S. Timóteo ${ }^{3}$ \\ ${ }^{1}$ Departamento de Cincias Exatas e Naturais, Universidade Estadual do Sudoeste da Bahia, Itapetinga, BA, Brazil \\ ${ }^{2}$ Centro de Rádio-Astronomia e Astrofísica, Universidade Presbiteriana Mackenzie, São Paulo, SP, Brazil \\ ${ }^{3}$ Faculdade de Tecnologia (FT), Universidade Estadual de Campinas (UNICAMP), Campinas, SP, Brazil
}

Correspondence should be addressed to V. S. Timóteo; varese@ft.unicamp.br

Received 3 November 2016; Revised 5 January 2017; Accepted 29 January 2017; Published 26 February 2017

Academic Editor: Mohammadreza Hadizadeh

Copyright (C) 2017 E. F. Batista et al. This is an open access article distributed under the Creative Commons Attribution License, which permits unrestricted use, distribution, and reproduction in any medium, provided the original work is properly cited. The publication of this article was funded by SCOAP $^{3}$.

We analyse the renormalization of the of two-nucleon interaction with multiple subtractions in peripheral waves considering two chiral forces at N3LO. Phase shifts at low energies are then computed with several subtraction points below $\mu=10 \mathrm{fm}^{-1}$. We show that for most peripheral waves the phase shifts have nearly no dependence on the renormalization scale. In two cases the phase shifts converge slowly as the renormalization scale approaches $\mu=1 \mathrm{fm}^{-1}$ and in one case the phase shifts presented oscillations with respect to the subtraction point $\mu$.

\section{Introduction}

It is widely established in nuclear physics that Quantum Chromodynamics (QCD) is in fact the underlying theory which describes the properties of strong nuclear forces. In this theory, the fundamental degrees of freedom are quarks which interact with each other via exchange of colored gluons. On the other hand, the strong nuclear force is also responsible for the binding of protons and neutrons in atomic nuclei. But according to QCD, nucleons are bound states of quarks and the nuclear force is considered as the residual part of the quark-quark interaction inside of the nucleon with exchange of gluons. Due to the property of asymptotic freedom, the running coupling constant is small enough at high energies to allow QCD to be handled within a perturbative approach. However, at low energies, where mesons and nucleons are the relevant degrees of freedom, the running coupling constant becomes large and QCD is no longer perturbative. The nonperturbative nature of QCD at low energies is implied in several mathematical and computational problems which makes the description of nucleon properties a hard task.

Since QCD cannot be treated perturbatively at low energies, a new approach was developed to handle nuclear forces with degrees of freedom appropriate for low-energy systems. The idea was to use quantum field theory but replacing quarks and gluons degrees of freedom by pions and nucleons, keeping the fundamental properties of the underlying theory like chiral symmetry. This effective field theory (EFT) scheme was already used in other systems to describe different types of interactions and Weinberg proposed an EFT approach to nuclear systems based on QCD. This idea generated a new branch in nuclear physics and allowed a deeper understanding of the nuclear force and few-nucleon systems. In particular, the two-nucleon system requires a nonperturbative extension of Chiral Perturbation Theory (ChPT) which works well in the case of pion-nucleon scattering.

Basically, an EFT is constructed by isolating the most relevant degrees of freedom and symmetries for the system under consideration and applying standard quantum field theory. In nuclear physics, replacing quarks and gluons by pions and nucleons means moving to a different (lower) energy scale and a connection between the symmetry properties of the underlying fundamental theory and the symmetries of their effective versions must be well-established. Thus, in a nuclear effective theories it is necessary to establish scale parameters which enable us to separate the high energy components of 
the interaction from the low-energy part. In a series of papers [1-3], Weinberg proposed an effective field theory scheme for nuclear forces based on the chiral symmetry of QCD.

This approach was first applied by Ordóñez et al. $[4,5]$ and allowed the perturbative treatment of the $N N$ interaction. An expansion in powers of $\left(Q / \Lambda_{\chi}\right)^{v}$ is performed and $Q$ is a generic low momentum scale and $\Lambda$ is the chiral symmetry breaking scale which is approximately $1 \mathrm{GeV}$. This expansion is controlled by a power counting scheme, called Weinberg power counting (WPC), which provides an hierarchical organization for the processes in few-nucleon systems. Following the WPC, the NN interaction at leading order (LO) consists of one-pion-exchange (OPE) plus a contact term. At next-toleading order (NLO), two-pion exchange (TPE) and $\mathcal{O}\left(p^{2}\right)$ contact interactions are added; at next-to-next-to-leading order (N2LO) there is an additional set of TPE diagrams and, finally, at next-to-next-to-next-to-leading order (N3LO) corrections to both OPE and TPE are included along with $\mathcal{O}\left(p^{4}\right)$ contact interactions.

Regardless of how the chiral expansion is organized, the issue of how to renormalize the two-body interaction is of fundamental importance and has been subject of investigation for decades. Early works by Adhikari et al. started by discussing the renormalization of two-body quantum hamiltonians [6-8]. Later, the problem was focused in the $N N$ interaction using renormalization group analysis and a new power counting was proposed by Kaplan et al. [9-11]. Discussions on the renormalization of singular and one-pion-exchange twobody interactions, power counting, and renormalization of the three-body system are detailed by van Kolck et al. [12-14]. Another renormalization group approach to two-body and nucleon-nucleon scattering was presented by Birse et al. [1518 ] and a complete analysis of cutoff renormalization in configuration space was performed by the Granada group [1922]. Also, a comparison between renormalization in configuration and momentum spaces has been carried out in [23].

Another renormalization approach for the $N N$ system consists of a hybrid scheme, where the LO contribution is treated nonperturbatively and the higher orders are handled perturbatively [24]. Results for $P$-waves and $D$-waves show that perturbative two-pion exchange reproduces the experimental data up to $k_{\mathrm{cm}} \sim 300 \mathrm{MeV}$. Here we treat all terms nonperturbatively since in our renormalization scheme the pieces of the interaction are inserted as the subtractions are performed.

Apart from the divergences due to pion loops in the irreducible diagrams, the reducible diagrams also generate divergences in the scattering equation. To overcome this problem the most common employed method is introducing a cutoff regularization scale $\Lambda$ which limits the momentum integration, in the scattering equation, above this scale parameter resulting in a finite phase shifts. The cutoff scheme handles the divergences by modifying the potential and keeping the scattering equation intact. The regularized interaction contains only low momentum components and the cutoff scale is fixed at some scale, typically $\sim 2-3 \mathrm{fm}^{-1}$. A slight discomfort with this method comes from the fact that all physics above a certain momentum scale is excluded. Recently, a N3LO interaction has been optimized by an improved renormalization approach in configuration space which maintains the analytic structure of the scattering amplitude [25].

An alternative renormalization procedure referred to as subtractive method or multiple subtractions treats the divergences with a different perspective: instead of modifying the potential, as in cutoff method, and keeping the scattering equation untouched, here the interaction is kept intact and the scattering equation is modified by the introduction of subtractions in its kernel. The dependence of the phase shifts on the cutoff is replaced by a dependence on the subtraction point that can be later eliminated by using the renormalization group flow. With this procedure no components are neglected from the interaction and both low and high momentum components are included. Detailed descriptions of this approach can be found in [26-32].

In this work we employ the subtract kernel method to renormalize the Lippmann-Schwinger equation in N3LO and perform a detailed renormalization scale dependence analysis of the phase shifts in peripheral channels. The high angular momentum waves are interesting because the force in their channels contains no contact interactions and consists only of pion exchanges. The N3LO potential has contact contributions up to $D$-waves so that $F$ and higher waves have contributions purely from pion exchanges and no core given by contact interactions.

This paper is organized as follows: Section 2 describes the renormalization of N3LO interactions with five subtractions; Section 3 presents the numerical results for both uncoupled and coupled channels up to $J=6$ and our main conclusions are given in Section 4.

\section{Renormalization of N3LO Interactions}

At any given order, the modern two-nucleon interactions can always be separated in two components: the pion exchange interactions and the contact terms, which parametrize the short range core of the interaction and are determined by fitting scattering data. The $N N$ potential is then written as

$$
V_{N N}=V_{\text {pions }}+V_{\text {cont }},
$$

where the first term contains one-pion-exchange and twopion exchanges

$$
V_{\text {pions }}=V_{\pi}+V_{2 \pi} .
$$

The power counting scheme organizes which set of Feynman diagrams must be included in each order in the chiral expansion:

$$
\begin{aligned}
& V_{\text {pions }}=V_{\text {pions }}^{(0)}+V_{\text {pions }}^{(2)}+V_{\text {pions }}^{(3)}+V_{\text {pions }}^{(4)}+\cdots, \\
& V_{\text {cont }}=V_{\text {cont }}^{(0)}+V_{\text {cont }}^{(2)}+V_{\text {cont }}^{(4)}+\cdots,
\end{aligned}
$$

where the superscript numbers in parentheses indicates the order in the chiral expansion. Note that in (3) for the contact contribution, all the odd powers are cancelled due to symmetry requirements. Hence, in the Weinberg power counting 
scheme, there is no contact interaction $V_{\text {cont }}^{(3)}$ at the third order in the chiral expansion (N2LO). This actually breaks the order-by-order improvement of the chiral expansion when going from NLO to N2LO as shown very clearly in [32]. Nogga et al. [14] looked at the interaction at leading order (LO) and found that additional counterterms that are not predicted by the Weinberg power counting are required in order to improve the description. Later, Valderrama showed that there are also problems in higher orders and they could be treated perturbatively [24].

For finite cutoff, however, Epelbaum et al. [33,34] showed that a better description of the phase shifts is obtained when N2LO instead of NLO interactions are used (with the same contact terms). The same conclusion can be found by studying $\chi^{2}$ /datum in Marji et al. [35].

Now let us turn to the renormalization of N3LO interactions with five subtractions. This approach introduces a renormalization scale $\mu$ (subtraction point), which denotes the momentum at which the subtractions are performed. For a given energy $E$ the Lippmann-Schwinger (LS) equation for the $T$-matrix in operator form is written as

$$
T(E)=V+V G_{0}^{+}(E) T(E),
$$

where $V$ is the $N N$ potential at a given order in the chiral expansion and $G_{0}^{+}$is the free Green's function which, in terms of the free Hamiltonian $H_{0}$, is given by

$$
G_{0}^{+}(E)=\frac{1}{E-H_{0}+i \epsilon} .
$$

When bare potentials are introduced in the equation above, an ultraviolet divergence arises due to the implicit integral in the second term of the right-hand side of (4), which diverges when the momentum goes to infinity. In the standard cutoff procedure, the $N N$ potential $V$ is multiplied by a regularizing function,

$$
\begin{aligned}
& V\left(p, p^{\prime}\right) \longrightarrow \\
& V_{\Lambda}\left(p, p^{\prime}\right) \\
& \quad \equiv \exp \left[-\left(\frac{p}{\Lambda}\right)^{2 r}\right] V\left(p, p^{\prime}\right) \exp \left[-\left(\frac{p^{\prime}}{\Lambda}\right)^{2 r}\right],
\end{aligned}
$$

where $\Lambda$ is the cutoff scale and $r \geq 1$. This function suppresses contributions from larger momenta, eliminating the ultraviolet divergences in the momentum integral. Nonrelativistic nucleon-nucleon potentials based on chiral effective field theory with cutoff regularization provide a very accurate description of $N N$ scattering data below pion production threshold $E_{\text {lab }} \sim 350 \mathrm{MeV}$.

The renormalization with multiple subtractions handles this problem in a different way since the NN potential is not modified in favour of changing Green's function instead. The N3LO interactions require five subtractions to be renormalized with no cutoff and in this case the subtracted scattering equation is given by

$$
T_{\mu}^{(5)}(E)=V_{\mu}^{(5)}(E)+V_{\mu}^{(5)}(E) G_{5}^{+}\left(E ;-\mu^{2}\right) T_{\mu}^{(5)}(E),
$$

where $\mu$ is the subtraction scale, $V_{\mu}^{(n)}(E)$ is the driving term

$$
\begin{aligned}
V_{\mu}^{(n)}(E)= & V_{\mu}^{(n-1)}(E) \\
& +V_{\mu}^{(n-1)}(E) \frac{\left(-\mu^{2}-E\right)^{n-1}}{\left(E-q^{2}\right)^{n}} V_{\mu}^{(n)}(E),
\end{aligned}
$$

which has to be calculated recursively, $G_{5}^{+}\left(E ;-\mu^{2}\right)$ is the 5 -times subtracted Green's function, and $q$ is the relative intermediate two-nucleon momentum.

$$
G_{5}^{+}(E)=\mathscr{F}_{5}\left(E ;-\mu^{2}\right) G_{0}^{+}(E),
$$

where

$$
\mathscr{F}_{5}\left(E ;-\mu^{2}\right)=\left(\frac{\mu^{2}+E}{\mu^{2}+H_{0}}\right)^{5}
$$

is a term that arises from the recursive nature of the renormalization process and works as a form factor, being responsible for providing a regular $T$-matrix. Detailed expressions for the integral equations in the recursive calculation with partial wave basis are given in [32] for the case of N2LO interactions with four subtractions.

The LS equation with five subtractions (7) has the same operator structure as the original equation (4), with the effective $N N$ potential $V$ replaced by the driving term $V_{\mu}^{(5)}(E)$ and the free Green's function $G_{0}^{+}(E)$ replaced by Green's function with five subtractions $G_{5}^{+}\left(E ;-\mu^{2}\right)$. The recursive driving term encodes the physical information apparently lost due to the removal of the propagation through intermediate states at the subtraction point $\mu$. Then, once the driving term is determined for a particular subtraction point, the subtracted Lippmann-Schwinger equation provides a renormalized solution for the $T$-matrix at any given energy $E$.

The driving terms $V_{\mu}^{(n)}$ are built recursively with the components of the nucleon-nucleon interaction. Here we use nonregulated N3LO interactions from Entem and Machleidt (EM) $[36,37]$ and from Epelbaum et al. (EGM) [38-40]. The main difference between the two chiral forces is the two-pion exchange part. The EGM potential uses Spectral Function Regularization (SFR) for the pion loop integrals resulting in a softer TPE component. Differences in the pionic part will then be compensated by changes in the low-energy constants so that in the end the two forces give similar descriptions for the NN system. Note that in the case of the EGM potential the SFR is still present for the loop integrals, but there is no smooth regulator function to suppress large momentum contributions. The smooth cutoff is also removed from the EM potential so that the interactions we are using are the original EM and EGM interactions with their cutoffs removed. Once the contact interactions are determined for both potentials, even with different off-shell behavior, the resulting on-shell scattering amplitudes are similar. Thus, we expect comparable results for cutoff regularization and subtractive renormalization at low energies. At high energies, $E_{\text {lab }}>200 \mathrm{MeV}$ the cutoff scheme is more efficient than the renormalization with multiple subtractions as far as describing the phase shifts is concerned. 

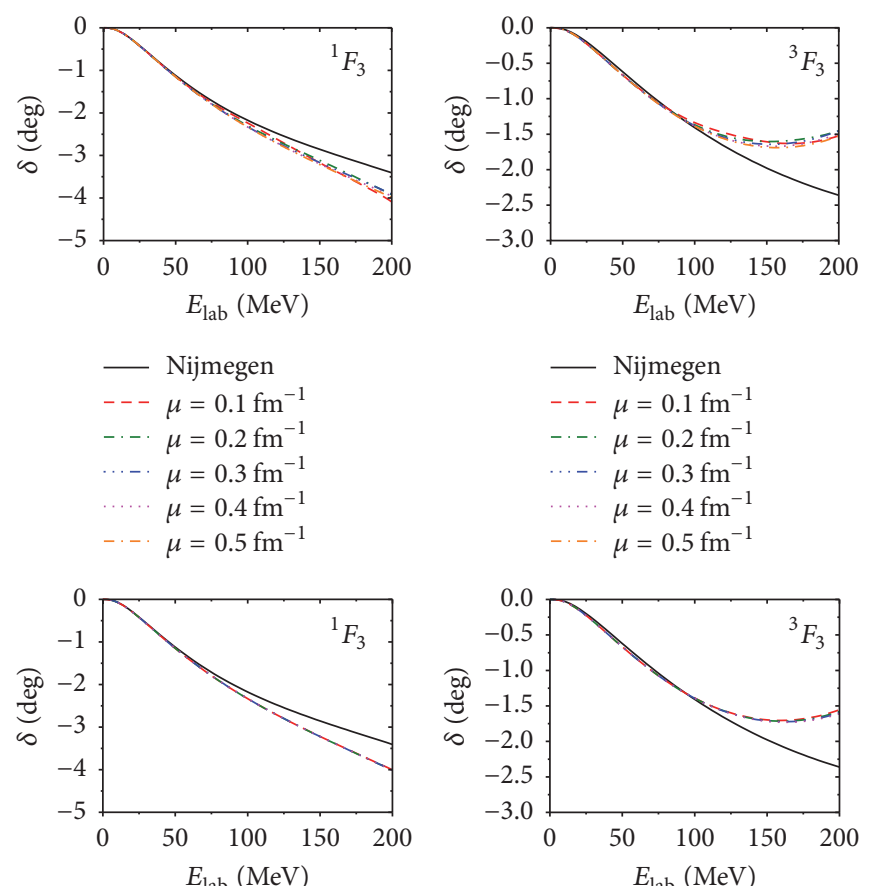

$$
\begin{array}{ll}
- & \text { Nijmegen } \\
--- & \mu=0.1 \mathrm{fm}^{-1} \\
-\cdot- & \mu=0.2 \mathrm{fm}^{-1} \\
\cdots- & \mu=0.3 \mathrm{fm}^{-1} \\
\cdots & \mu=0.4 \mathrm{fm}^{-1} \\
\cdots-\cdot & \mu=0.5 \mathrm{fm}^{-1}
\end{array}
$$

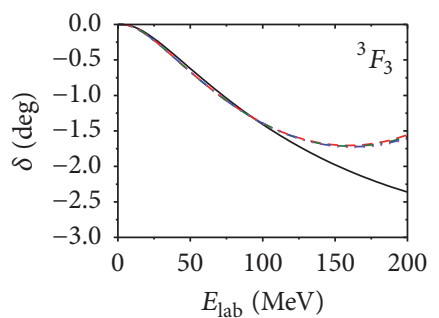

$$
\begin{aligned}
& \text { — Nijmegen } \\
& -\cdots \mu=0.6 \mathrm{fm}^{-1} \\
& \cdots \cdots=0.7 \mathrm{fm}^{-1} \\
& \cdots-\mu=0.8 \mathrm{fm}^{-1} \\
& \cdots \cdots
\end{aligned}
$$
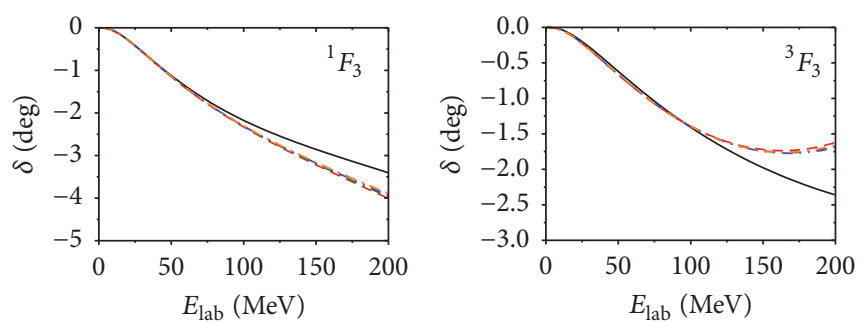

$$
\begin{aligned}
& \text { - Nijmegen } \\
& \text { - - } \mu=1.0 \mathrm{fm}^{-1} \\
& \text {-. } \mu=2.0 \mathrm{fm}^{-1} \\
& \cdots \mu=3.0 \mathrm{fm}^{-1} \\
& \text { … } \mu=4.0 \mathrm{fm}^{-1} \\
& \text { ‥ } \mu=5.0 \mathrm{fm}^{-1}
\end{aligned}
$$

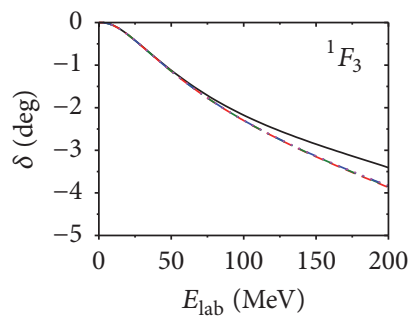

$$
\begin{array}{ll}
\text { - Nijmegen } \\
\cdots & \mu=6.0 \mathrm{fm}^{-1} \\
\cdots \cdots & \mu=7.0 \mathrm{fm}^{-1} \\
\cdots- & \mu=8.0 \mathrm{fm}^{-1} \\
\cdots \cdots & \mu=9.0 \mathrm{fm}^{-1}
\end{array}
$$

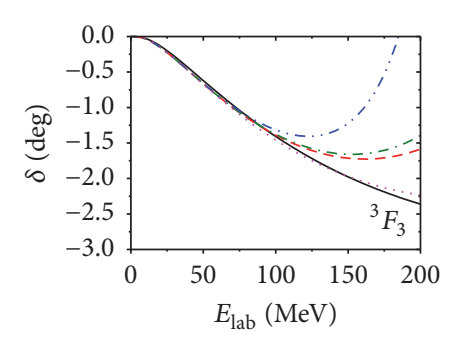

$$
\begin{aligned}
& \text { - Nijmegen } \\
& \text { - - } \mu=1.0 \mathrm{fm}^{-1} \\
& \text {-. } \mu=2.0 \mathrm{fm}^{-1} \\
& \text { ‥ } \mu=3.0 \mathrm{fm}^{-1} \\
& \text { … } \mu=4.0 \mathrm{fm}^{-1} \\
& \text {-.. } \mu=5.0 \mathrm{fm}^{-1}
\end{aligned}
$$

$$
\begin{aligned}
& \text { - Nijmegen } \\
& \cdots \mu=7.0 \mathrm{fm}^{-1} \\
& \text { … } \mu=8.0 \mathrm{fm}^{-1} \\
& \mu=9.0 \mathrm{fm}^{-1}
\end{aligned}
$$

Figure 1: (Color on-line) phase shifts in ${ }^{1} F_{3}$ and ${ }^{3} F_{3}$ uncoupled channels calculated from the solution of the subtracted LS equation for the $K$-matrix with five subtractions for the N3LO-EM potential for several values of the renormalization scale compared to the Nijmegen partial wave analysis. 

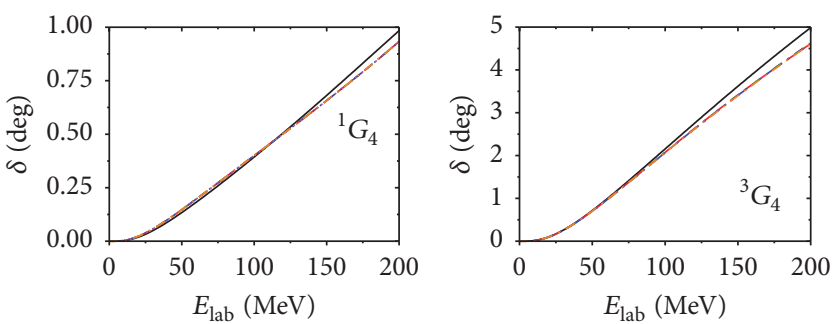

$$
\begin{array}{ll}
- & \text { Nijmegen } \\
\cdots-- & \mu=0.1 \mathrm{fm}^{-1} \\
\cdots- & \mu=0.2 \mathrm{fm}^{-1} \\
\cdots- & \mu=0.3 \mathrm{fm}^{-1} \\
\cdots \cdots & \mu=0.4 \mathrm{fm}^{-1} \\
\cdots & \mu=0.5 \mathrm{fm}^{-1}
\end{array}
$$

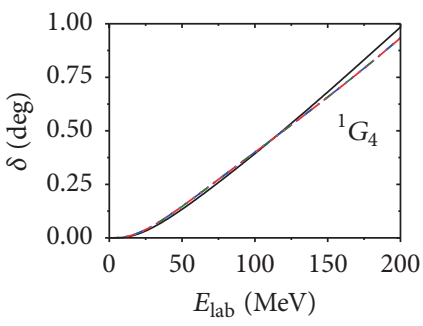

$$
\begin{aligned}
& \text { - Nijmegen } \\
& ---\mu=0.6 \mathrm{fm}^{-1} \\
& -\cdots \mu=0.7 \mathrm{fm}^{-1} \\
& \cdots-\mu=0.8 \mathrm{fm}^{-1} \\
& \cdots \cdots=0.9 \mathrm{fm}^{-1}
\end{aligned}
$$

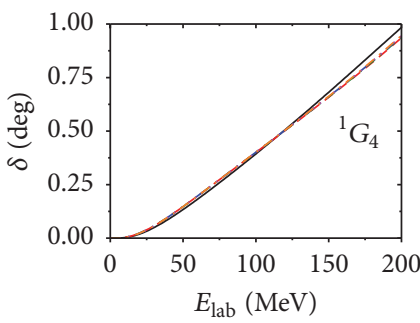

$$
\begin{aligned}
& \text { - Nijmegen } \\
& --\mu=1.0 \mathrm{fm}^{-1} \\
& \text {-.. } \mu=2.0 \mathrm{fm}^{-1} \\
& \text { … } \mu=3.0 \mathrm{fm}^{-1} \\
& \text { … } \mu=4.0 \mathrm{fm}^{-1} \\
& \mu=5.0 \mathrm{fm}^{-1}
\end{aligned}
$$

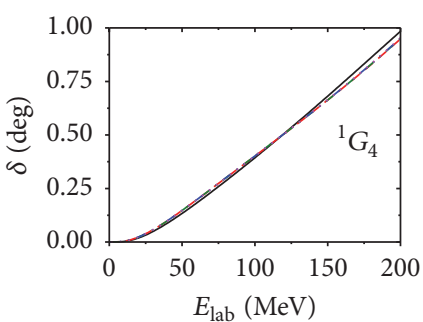

$$
\begin{aligned}
& \text { - Nijmegen } \\
& \text { - - } \mu=6.0 \mathrm{fm}^{-1} \\
& \text {-.. } \mu=7.0 \mathrm{fm}^{-1} \\
& \text { … } \mu=8.0 \mathrm{fm}^{-1} \\
& \mu=9.0 \mathrm{fm}^{-1}
\end{aligned}
$$

- Nijmegen

$---\mu=0.1 \mathrm{fm}^{-1}$

-.. $\mu=0.2 \mathrm{fm}^{-1}$

$\cdots-\mu=0.3 \mathrm{fm}^{-1}$

… $\mu=0.4 \mathrm{fm}^{-1}$

$\mu=0.5 \mathrm{fm}^{-1}$

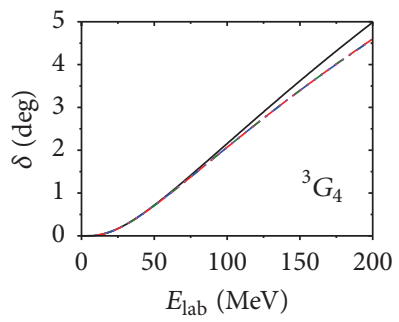

- Nijmegen

- - $\mu=0.6 \mathrm{fm}^{-1}$

$\cdots \mu=0.7 \mathrm{fm}^{-1}$

… $\mu=0.8 \mathrm{fm}^{-1}$

$\mu=0.9 \mathrm{fm}^{-1}$

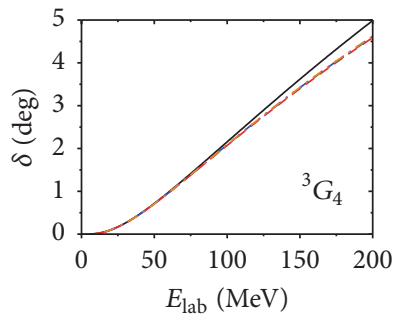

— Nijmegen

$--\mu=1.0 \mathrm{fm}^{-1}$

-. $\mu=2.0 \mathrm{fm}^{-1}$

… $\mu=3.0 \mathrm{fm}^{-1}$

…. $\mu=4.0 \mathrm{fm}^{-1}$

$\mu=5.0 \mathrm{fm}^{-1}$

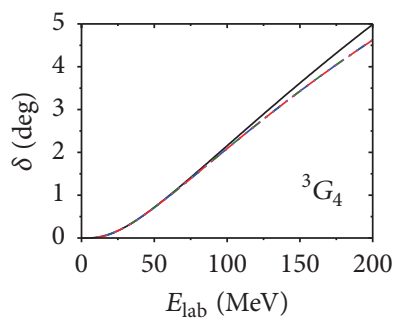

- Nijmegen

$---\mu=6.0 \mathrm{fm}^{-1}$

-.. $\mu=7.0 \mathrm{fm}^{-1}$

… $\mu=8.0 \mathrm{fm}^{-1}$

…. $\mu=9.0 \mathrm{fm}^{-1}$

Figure 2: (Color on-line) phase shifts in ${ }^{1} G_{4}$ and ${ }^{3} G_{4}$ uncoupled channels calculated from the solution of the subtracted LS equation for the $K$-matrix with five subtractions for the N3LO-EM potential for several values of the renormalization scale compared to the Nijmegen partial wave analysis. 

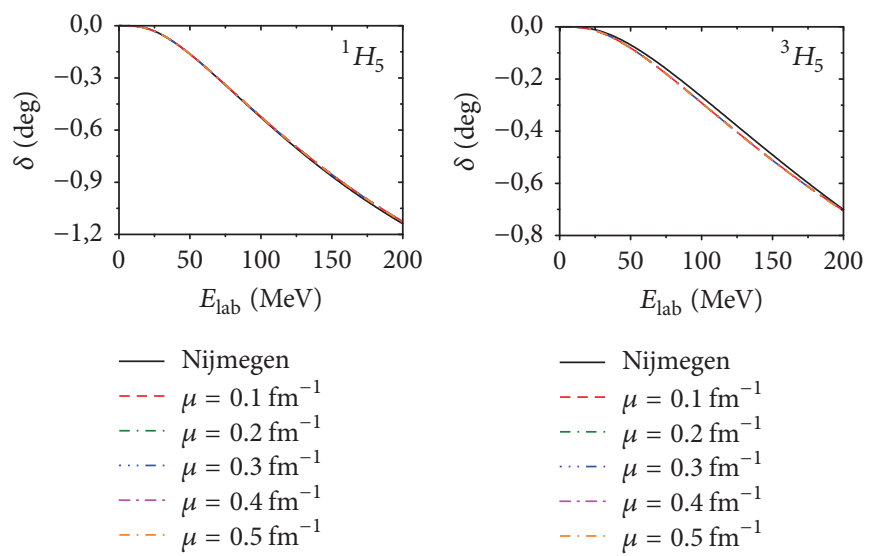

- Nijmegen

- - $\mu=0.1 \mathrm{fm}^{-1}$

-. $\mu=0.2 \mathrm{fm}^{-1}$

‥ $\mu=0.3 \mathrm{fm}^{-1}$

$\cdots \mu=0.4 \mathrm{fm}^{-1}$

-. $\mu=0.5 \mathrm{fm}^{-1}$
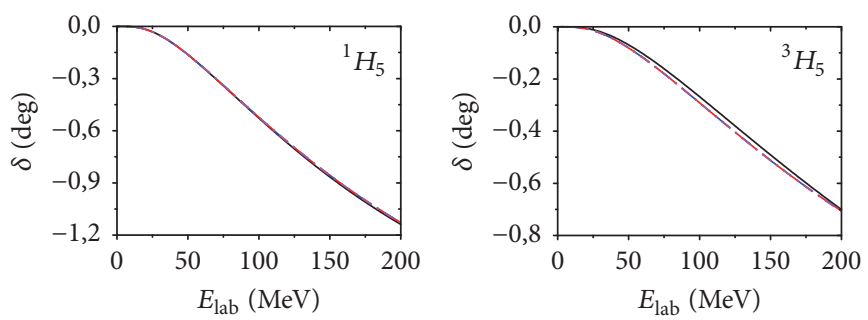

$\begin{array}{ll}- & \text { Nijmegen } \\ --- & \mu=0.6 \mathrm{fm}^{-1} \\ -\cdots & \mu=0.7 \mathrm{fm}^{-1} \\ \cdots- & \mu=0.8 \mathrm{fm}^{-1} \\ \cdots & \mu=0.9 \mathrm{fm}^{-1}\end{array}$

- Nijmegen

- - $\mu=0.6 \mathrm{fm}^{-1}$

-. . $\mu=0.7 \mathrm{fm}^{-1}$

$\cdots-\mu=0.8 \mathrm{fm}^{-1}$

$\cdots \mu=0.9 \mathrm{fm}^{-1}$
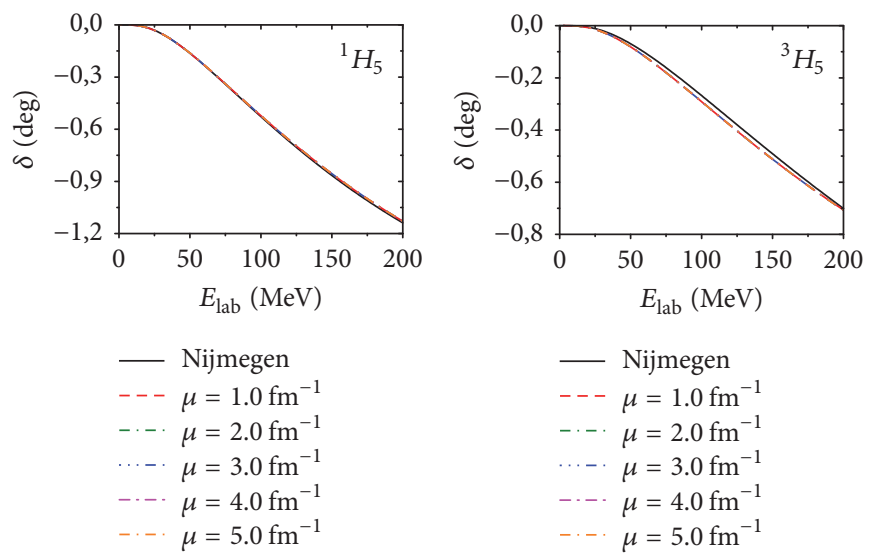

- Nijmegen

- - $\mu=1.0 \mathrm{fm}^{-1}$

-.. $\mu=2.0 \mathrm{fm}^{-1}$

$\cdots-\mu=3.0 \mathrm{fm}^{-1}$

-.. $\mu=4.0 \mathrm{fm}^{-1}$

-.. $\mu=5.0 \mathrm{fm}^{-1}$
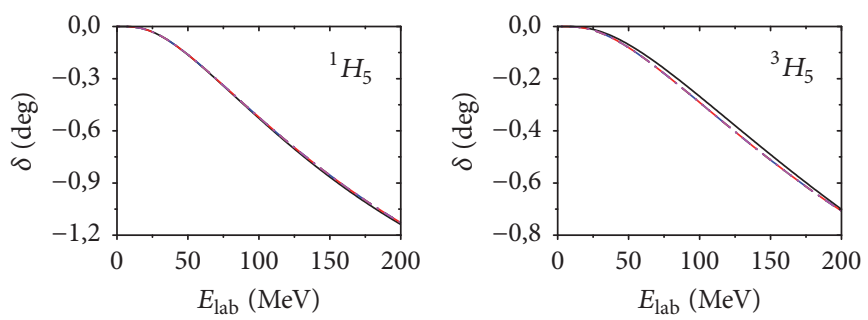

- Nijmegen
$---\mu=6.0 \mathrm{fm}^{-1}$
$-\cdot-\mu=7.0 \mathrm{fm}^{-1}$
$\cdots-\mu=8.0 \mathrm{fm}^{-1}$
$-\cdots$

- Nijmegen

- - $\mu=6.0 \mathrm{fm}^{-1}$

-.. $\mu=7.0 \mathrm{fm}^{-1}$

… $\mu=8.0 \mathrm{fm}^{-1}$

$\cdots \mu=9.0 \mathrm{fm}^{-1}$

Figure 3: (Color on-line) phase shifts in ${ }^{1} \mathrm{H}_{5}$ and ${ }^{3} \mathrm{H}_{5}$ uncoupled channels calculated from the solution of the subtracted LS equation for the $K$-matrix with five subtractions for the N3LO-EM potential for several values of the renormalization scale compared to the Nijmegen partial wave analysis. 

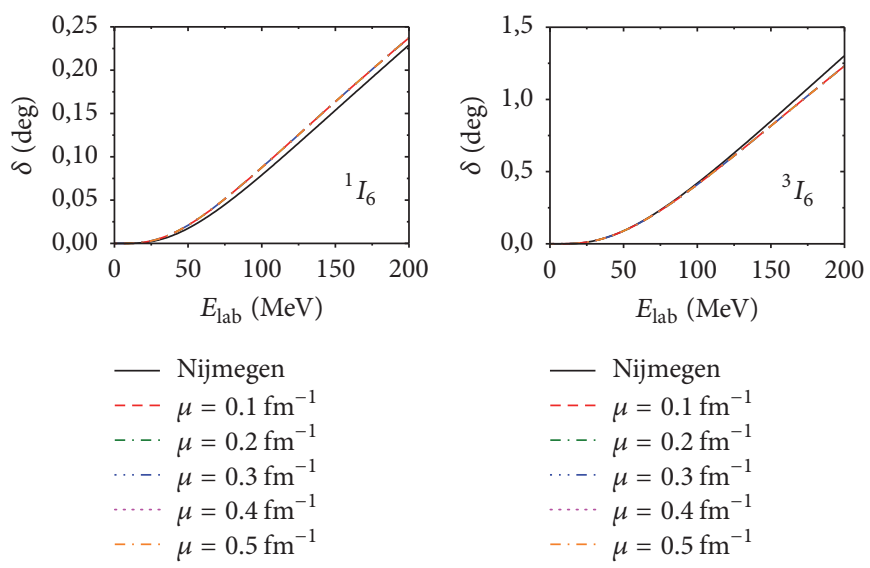

$$
\begin{aligned}
& \text { - Nijmegen } \\
& \text { - - } \mu=0.1 \mathrm{fm}^{-1} \\
& \text {-.. } \mu=0.2 \mathrm{fm}^{-1} \\
& \text { … } \mu=0.3 \mathrm{fm}^{-1} \\
& \text { … } \mu=0.4 \mathrm{fm}^{-1} \\
& \text {-.. } \mu=0.5 \mathrm{fm}^{-1}
\end{aligned}
$$
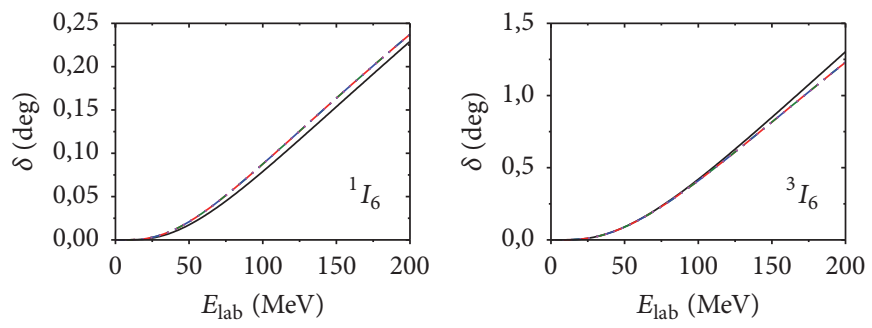

$$
\begin{aligned}
& \text { Nijmegen } \\
& ---\mu=0.6 \mathrm{fm}^{-1} \\
& -\cdot-\mu=0.7 \mathrm{fm}^{-1} \\
& \cdots-\cdot \mu=0.8 \mathrm{fm}^{-1} \\
& \cdots \cdots \cdot \mu=0.9 \mathrm{fm}^{-1}
\end{aligned}
$$
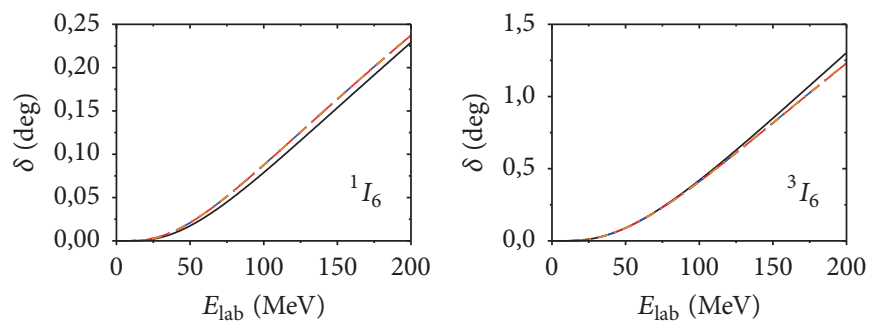

$$
\begin{aligned}
& \text { - Nijmegen } \\
& \text { - - } \mu=1.0 \mathrm{fm}^{-1} \\
& \text {-.. } \mu=2.0 \mathrm{fm}^{-1} \\
& \text { ‥ } \mu=3.0 \mathrm{fm}^{-1} \\
& \mu=4.0 \mathrm{fm}^{-1} \\
& \mu=5.0 \mathrm{fm}^{-1}
\end{aligned}
$$

$$
\begin{aligned}
& - \text { Nijmegen } \\
& -\cdots \mu=0.6 \mathrm{fm}^{-1} \\
& \cdots \cdots=0.7 \mathrm{fm}^{-1} \\
& \cdots-\mu=0.8 \mathrm{fm}^{-1} \\
& \cdots \cdots
\end{aligned}
$$

$$
\begin{aligned}
& \text { - Nijmegen } \\
& \text { - - } \mu=1.0 \mathrm{fm}^{-1} \\
& \text {-.. } \mu=2.0 \mathrm{fm}^{-1} \\
& \cdots-\mu=3.0 \mathrm{fm}^{-1} \\
& \mu=4.0 \mathrm{fm}^{-1} \\
& \mu=5.0 \mathrm{fm}^{-1}
\end{aligned}
$$
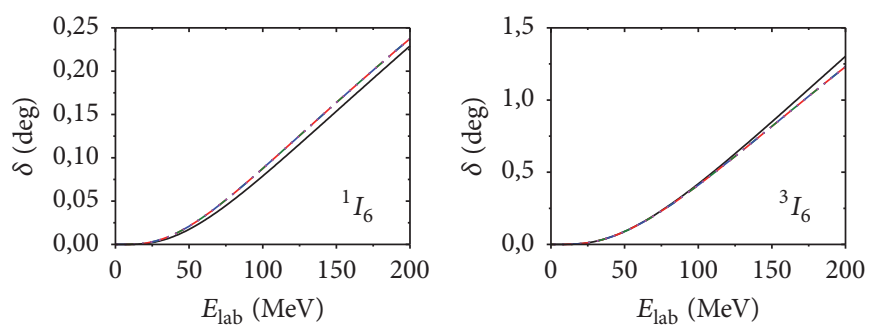

$$
\begin{aligned}
& \text { - Nijmegen } \\
& \text { - - } \mu=6.0 \mathrm{fm}^{-1} \\
& \text {-.. } \mu=7.0 \mathrm{fm}^{-1} \\
& \text { ‥ } \mu=8.0 \mathrm{fm}^{-1} \\
& \mu=9.0 \mathrm{fm}^{-1}
\end{aligned}
$$

$$
\begin{aligned}
& \text { _ Nijmegen } \\
& \text { - - } \mu=6.0 \mathrm{fm}^{-1} \\
& \text {-.. } \mu=7.0 \mathrm{fm}^{-1} \\
& \text { … } \mu=8.0 \mathrm{fm}^{-1} \\
& \mu=9.0 \mathrm{fm}^{-1}
\end{aligned}
$$

Figure 4: (Color on-line) phase shifts in ${ }^{1} I_{6}$ and ${ }^{3} I_{6}$ uncoupled channels calculated from the solution of the subtracted LS equation for the $K$-matrix with five subtractions for the N3LO-EM potential for several values of the renormalization scale compared to the Nijmegen partial wave analysis. 

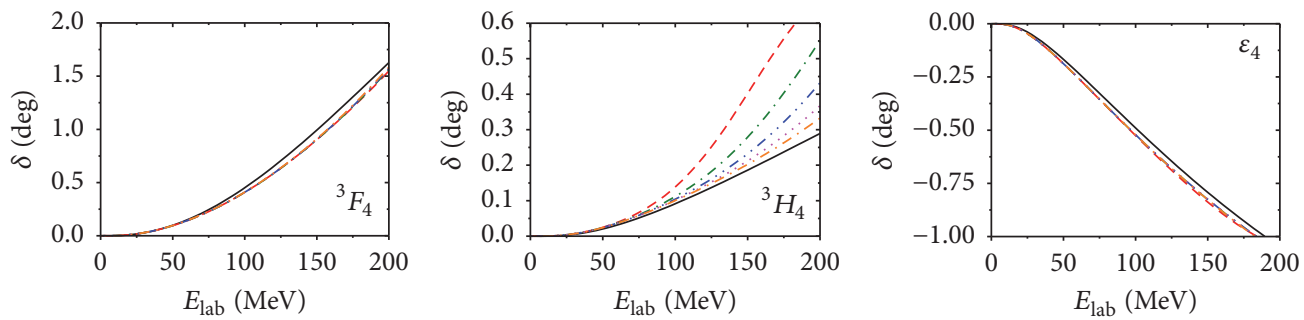

$$
\begin{aligned}
& \text { - Nijmegen } \\
& \text { - - } \mu=0.1 \mathrm{fm}^{-1} \\
& \text {-. } \mu=0.2 \mathrm{fm}^{-1} \\
& \text { … } \mu=0.3 \mathrm{fm}^{-1} \\
& \text { … } \mu=0.4 \mathrm{fm}^{-1} \\
& \text {-.. } \mu=0.5 \mathrm{fm}^{-1}
\end{aligned}
$$

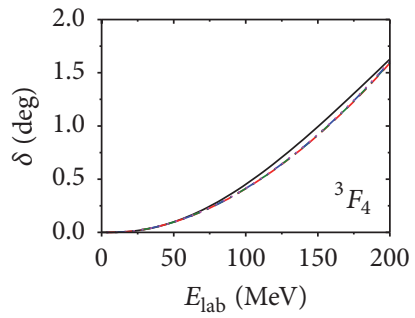

$$
\begin{aligned}
& \text { - Nijmegen } \\
& \cdots \mu=0.6 \mathrm{fm}^{-1} \\
& \cdots \mu=0.7 \mathrm{fm}^{-1} \\
& \cdots \cdots=0.8 \mathrm{fm}^{-1} \\
& \cdots \cdots
\end{aligned}
$$

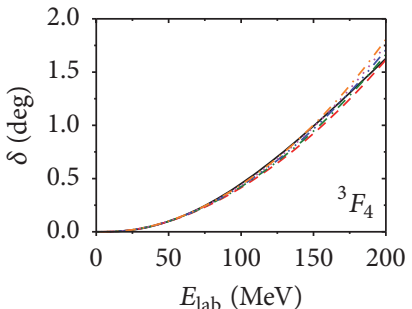

$$
\begin{aligned}
& \text { _ Nijmegen } \\
& \text { - - } \mu=1.0 \mathrm{fm}^{-1} \\
& \cdots \mu=2.0 \mathrm{fm}^{-1} \\
& \text { … } \mu=3.0 \mathrm{fm}^{-1} \\
& \text { … } \mu=4.0 \mathrm{fm}^{-1} \\
& \mu=5.0 \mathrm{fm}^{-1}
\end{aligned}
$$

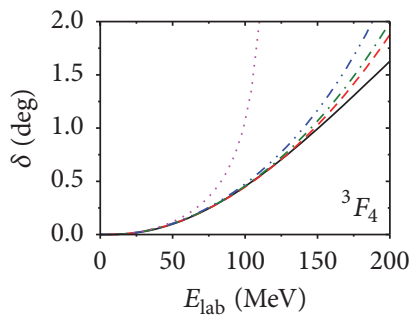

$$
\begin{aligned}
& \text { - Nijmegen } \\
& \text { - - } \mu=6.0 \mathrm{fm}^{-1} \\
& \text {-. } \mu=7.0 \mathrm{fm}^{-1} \\
& \text { … } \mu=8.0 \mathrm{fm}^{-1} \\
& \mu=9.0 \mathrm{fm}^{-1}
\end{aligned}
$$

$$
\begin{aligned}
& \text { _ Nijmegen } \\
& \text { - - } \mu=0.1 \mathrm{fm}^{-1} \\
& \text {-.. } \mu=0.2 \mathrm{fm}^{-1} \\
& \mu=0.3 \mathrm{fm}^{-1} \\
& \text { … } \mu=0.4 \mathrm{fm}^{-1} \\
& \text {-.. } \mu=0.5 \mathrm{fm}^{-1}
\end{aligned}
$$

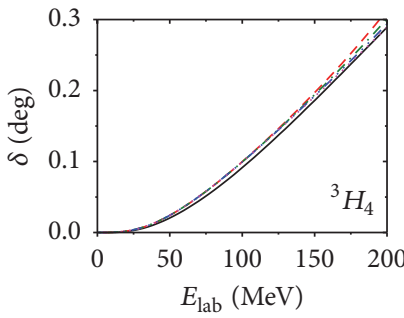

$$
\begin{aligned}
& \text { Nijmegen } \\
& -\cdots \mu=0.6 \mathrm{fm}^{-1} \\
& \cdots \cdots=0.7 \mathrm{fm}^{-1} \\
& \cdots \cdots=0.8 \mathrm{fm}^{-1} \\
& \cdots \cdots \\
& \mu=0.9 \mathrm{fm}^{-1}
\end{aligned}
$$

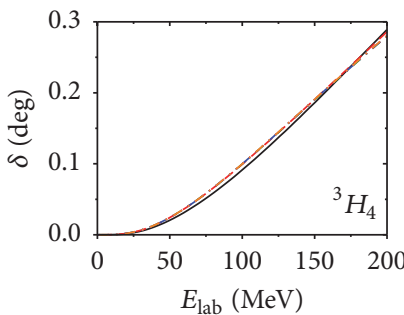

$$
\begin{aligned}
& \ldots \text { Nijmegen } \\
& -\cdots \mu=1.0 \mathrm{fm}^{-1} \\
& \cdots \cdots=2.0 \mathrm{fm}^{-1} \\
& \cdots-\mu=3.0 \mathrm{fm}^{-1} \\
& \cdots \cdots=4.0 \mathrm{fm}^{-1} \\
& \cdots \quad \mu=5.0 \mathrm{fm}^{-1}
\end{aligned}
$$

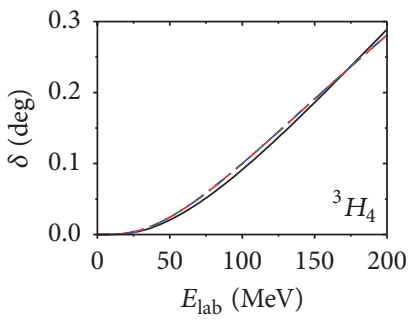

$$
\begin{aligned}
& \text { Nijmegen } \\
& -\cdots \mu=6.0 \mathrm{fm}^{-1} \\
& \cdots \cdots=7.0 \mathrm{fm}^{-1} \\
& \cdots-\mu=8.0 \mathrm{fm}^{-1} \\
& \cdots \quad \mu=9.0 \mathrm{fm}^{-1}
\end{aligned}
$$

_ Nijmegen

- - $\mu=0.1 \mathrm{fm}^{-1}$

-. $\mu=0.2 \mathrm{fm}^{-1}$

… $\mu=0.3 \mathrm{fm}^{-1}$

… $\mu=0.4 \mathrm{fm}^{-1}$

-.. $\mu=0.5 \mathrm{fm}^{-1}$

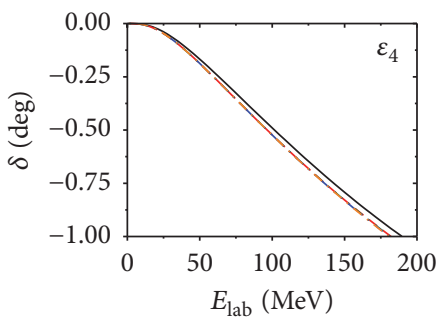

- Nijmegen

$---\mu=0.6 \mathrm{fm}^{-1}$

-.. $\mu=0.7 \mathrm{fm}^{-1}$

… $\mu=0.8 \mathrm{fm}^{-1}$

$\cdots \mu=0.9 \mathrm{fm}^{-1}$

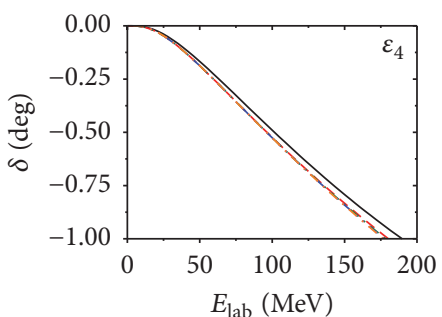

- Nijmegen

- - $\mu=1.0 \mathrm{fm}^{-1}$

$\cdots \mu=2.0 \mathrm{fm}^{-1}$

… $\mu=3.0 \mathrm{fm}^{-1}$

… $\mu=4.0 \mathrm{fm}^{-1}$

-.. $\mu=5.0 \mathrm{fm}^{-1}$

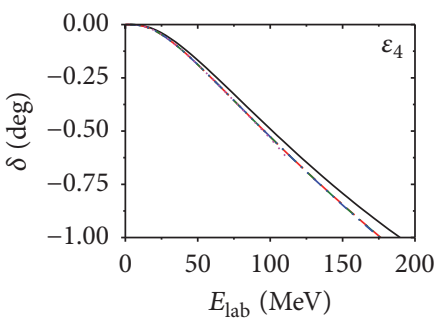

$$
\begin{aligned}
& \text { - Nijmegen } \\
& \text { - - } \mu=6.0 \mathrm{fm}^{-1} \\
& \text {-. } \mu=7.0 \mathrm{fm}^{-1} \\
& \text { … } \mu=8.0 \mathrm{fm}^{-1} \\
& \mu=9.0 \mathrm{fm}^{-1}
\end{aligned}
$$

Figure 5: (Color on-line) phase shifts in ${ }^{3} F_{4}-{ }^{3} H_{4}$ coupled channels calculated from the solution of the subtracted LS equation for the $K$-matrix with five subtractions for the N3LO-EM potential for several values of the renormalization scale compared to the Nijmegen partial wave analysis. 

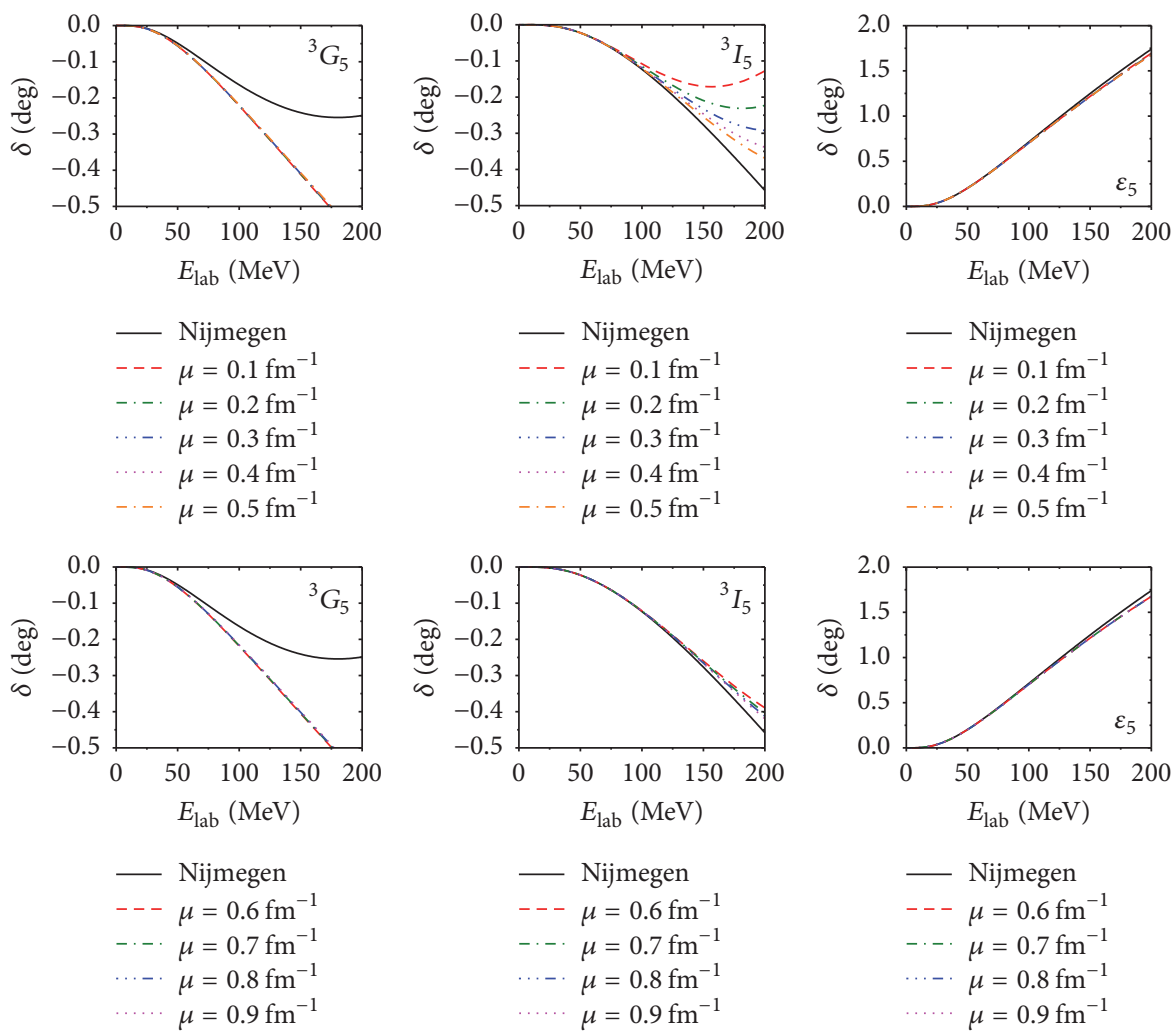

- Nijmegen

- - $\mu=0.6 \mathrm{fm}^{-1}$

-. $\mu=0.7 \mathrm{fm}^{-1}$

‥ $\mu=0.8 \mathrm{fm}^{-1}$

… $\mu=0.9 \mathrm{fm}^{-1}$
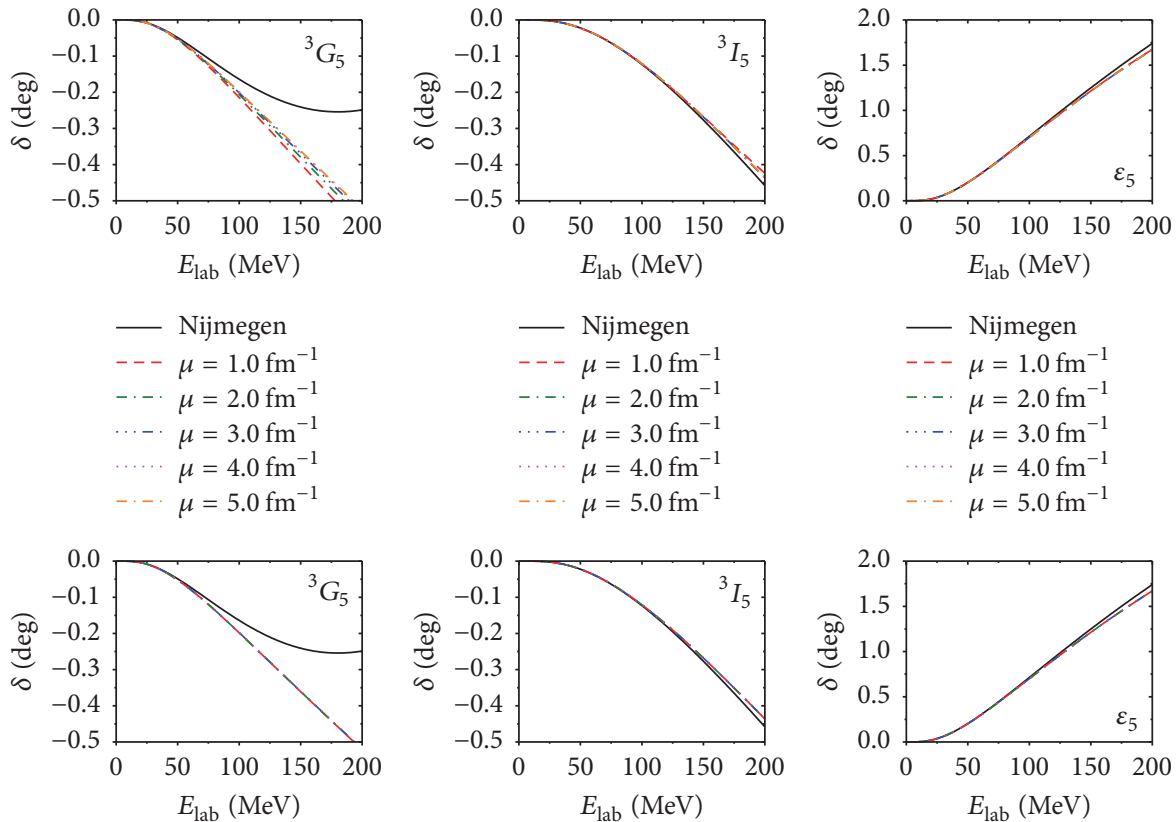

— Nijmegen

- - $\mu=1.0 \mathrm{fm}^{-1}$

-. . $\mu=2.0 \mathrm{fm}^{-1}$

‥ $\mu=3.0 \mathrm{fm}^{-1}$ $\mu=4.0 \mathrm{fm}^{-1}$

-.. $\mu=5.0 \mathrm{fm}^{-1}$
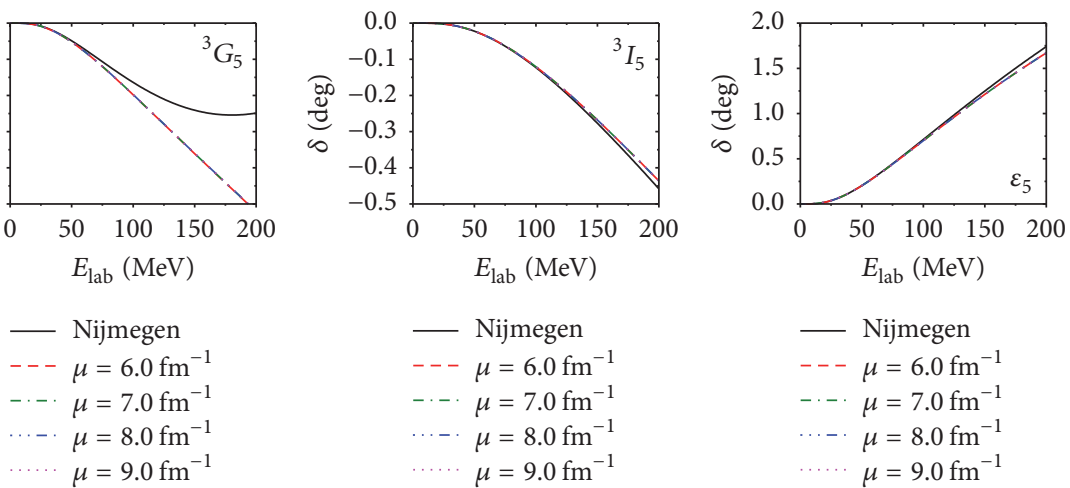

$$
\begin{aligned}
& \text { - Nijmegen } \\
& \text {-. } \mu=6.0 \mathrm{fm}^{-1} \\
& \text {-.- } \mu=7.0 \mathrm{fm}^{-1} \\
& \text { … } \mu=8.0 \mathrm{fm}^{-1} \\
& \mu=9.0 \mathrm{fm}^{-1}
\end{aligned}
$$

Figure 6: (Color on-line) phase shifts in ${ }^{3} G_{5}-{ }^{3} I_{5}$ coupled channels calculated from the solution of the subtracted LS equation for the $K$ matrix with five subtractions for the N3LO-EM potential for several values of the renormalization scale compared to the Nijmegen partial wave analysis. 

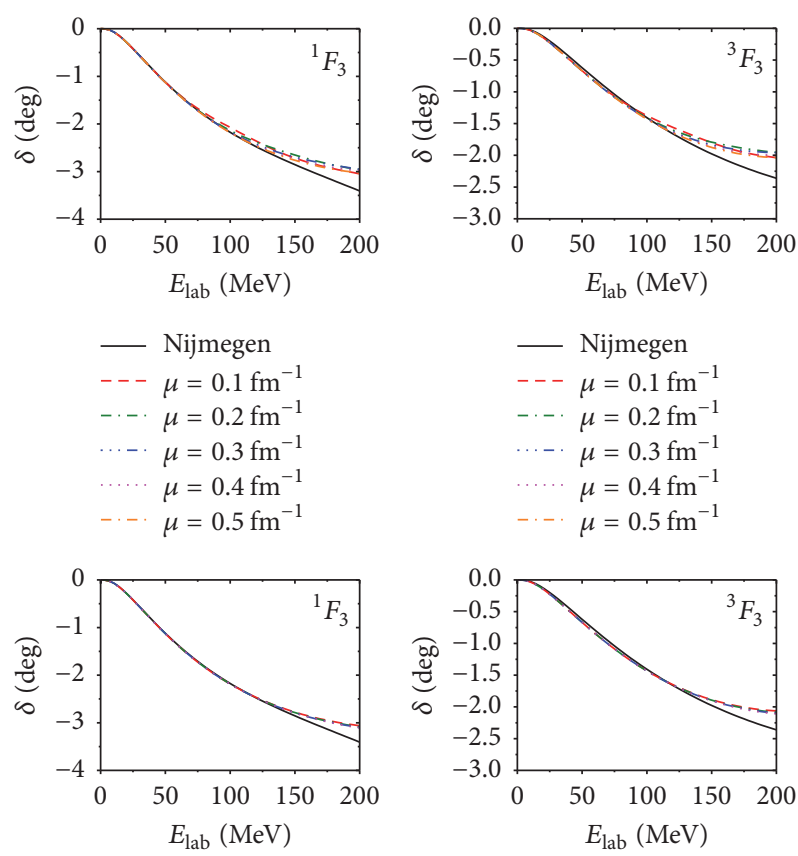

- Nijmegen

$---\mu=0.6 \mathrm{fm}^{-1}$

-.. $\mu=0.7 \mathrm{fm}^{-1}$

$\cdots-\mu=0.8 \mathrm{fm}^{-1}$

$\mu=0.9 \mathrm{fm}^{-1}$

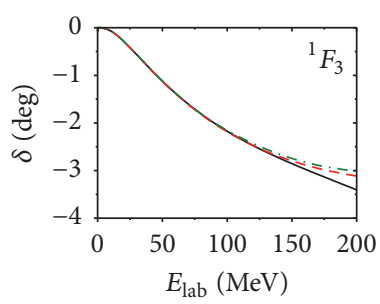

- Nijmegen

$--\mu=0.6 \mathrm{fm}^{-1}$

$\cdots \mu=0.7 \mathrm{fm}^{-1}$

… $\mu=0.8 \mathrm{fm}^{-1}$

$\mu=0.9 \mathrm{fm}^{-1}$

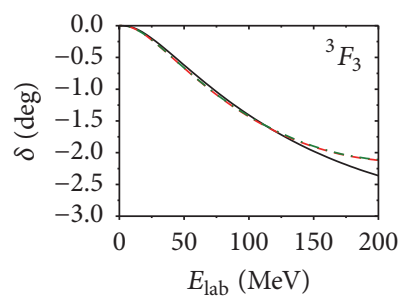

- Nijmegen

$--\mu=1.0 \mathrm{fm}^{-1}$

-.. $\mu=2.0 \mathrm{fm}^{-1}$

- Nijmegen
$--\mu=1.0 \mathrm{fm}^{-1}$
$-\cdots \mu=2.0 \mathrm{fm}^{-1}$

Figure 7: (Color on-line) phase shifts in ${ }^{1} F_{3}$ and ${ }^{3} F_{3}$ channels calculated from the solution of the subtracted LS equation for the $K$-matrix with five subtractions for the N3LO-EGM potential for several values of the renormalization scale compared to the Nijmegen partial wave analysis.

\section{Numerical Results}

Here we work in a partial wave relative momentum space basis and compute the phase shifts in each peripheral channel. However, a three-dimensional approach without any partial wave decomposition has also been employed [41-43].

For numerical reasons, when implementing the renormalization procedure, we solve the subtracted LS equation for the $K$-matrix using the principal value prescription. We then compute the neutron-proton phase shifts for channels with angular momentum in the range $3 \leq J \leq 6$ with the N3LO potentials EM and EGM. Expressions for the phases shits as
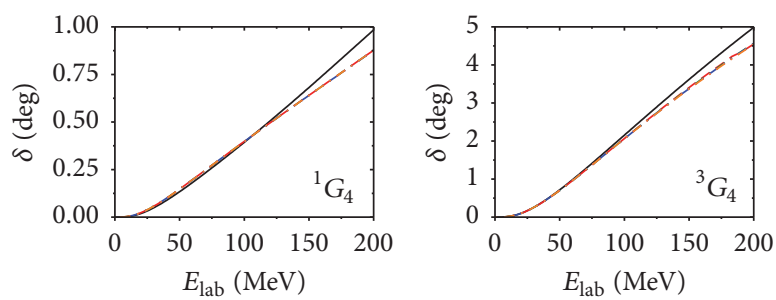

$$
\begin{array}{ll}
- & \text { Nijmegen } \\
--- & \mu=0.1 \mathrm{fm}^{-1} \\
\cdots-\cdot & \mu=0.2 \mathrm{fm}^{-1} \\
\cdots- & \mu=0.3 \mathrm{fm}^{-1} \\
\cdots \cdots & \mu=0.4 \mathrm{fm}^{-1} \\
\cdots-\cdot \mu & \mu=0.5 \mathrm{fm}^{-1}
\end{array}
$$
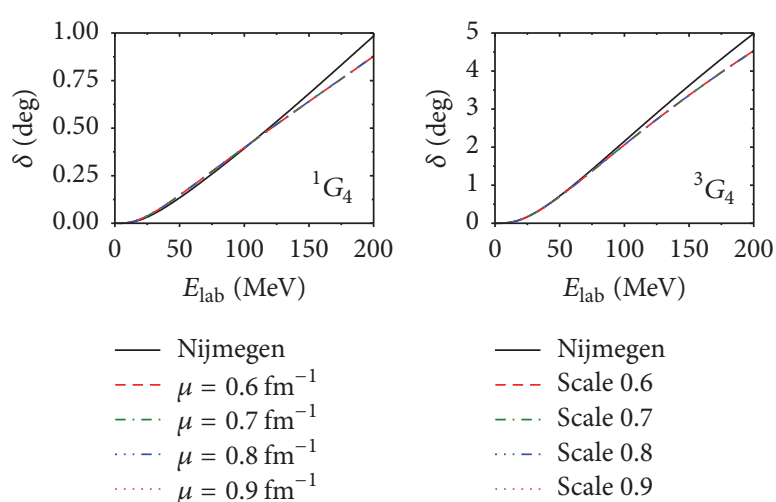

$$
\begin{aligned}
& \text { - Nijmegen } \\
& \text { - - } \mu=0.6 \mathrm{fm}^{-1} \\
& \mu=0.7 \mathrm{fm}^{-1} \\
& \text {-. } \mu=0.8 \mathrm{fm}^{-1} \\
& \mu=0.9 \mathrm{fm}^{-1}
\end{aligned}
$$
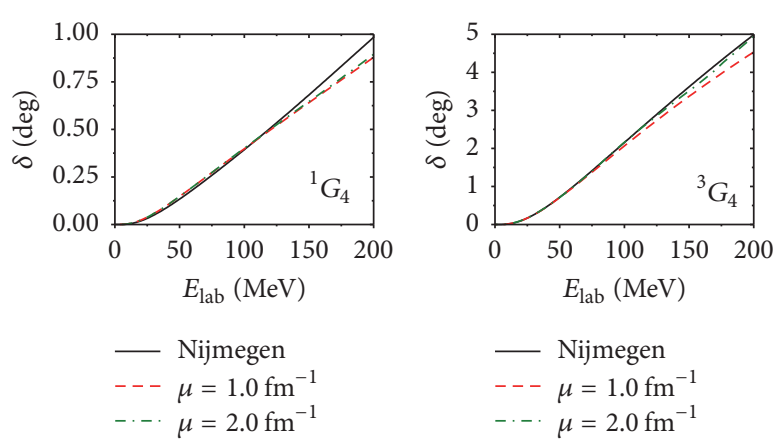

$$
\begin{aligned}
& \text { _ Nijmegen } \\
& \text { - - } \mu=1.0 \mathrm{fm}^{-1} \\
& \text {-.. } \mu=2.0 \mathrm{fm}^{-1}
\end{aligned}
$$

$$
\begin{array}{ll}
-\ldots & \text { Nijmegen } \\
-\cdots & \mu=0.1 \mathrm{fm}^{-1} \\
\cdots \cdots & \mu=0.2 \mathrm{fm}^{-1} \\
\cdots- & \mu=0.3 \mathrm{fm}^{-1} \\
\cdots \cdots & \mu=0.4 \mathrm{fm}^{-1} \\
\cdots-\cdots & \mu=0.5 \mathrm{fm}^{-1}
\end{array}
$$

Figure 8: (Color on-line) phase shifts in ${ }^{1} G_{4}$ and ${ }^{3} G_{4}$ channels calculated from the solution of the subtracted LS equation for the $K$-matrix with five subtractions for the N3LO-EGM potential for several values of the renormalization scale compared to the Nijmegen partial wave analysis.

functions of the on-shell $K$-matrix in coupled and uncoupled channels are given in [44-46].

For each wave, we consider several renormalization scales up to $\mu=10 \mathrm{fm}^{-1}$, limiting the momentum integrations at $\Lambda=30 \mathrm{fm}^{-1}$. In practice this means we have an infinite cutoff and the renormalization is completely imposed by the five subtractions, unlike in [47-49] where the cutoff still plays a role since only one subtraction was performed for the N2LO potential which requires four subtractions to allow an infinite cutoff.

In the case of the EGM potential, we have used an SFR cutoff of $\widetilde{\Lambda}=4 m_{\pi}(550 \mathrm{MeV})$, the most common choice. The 

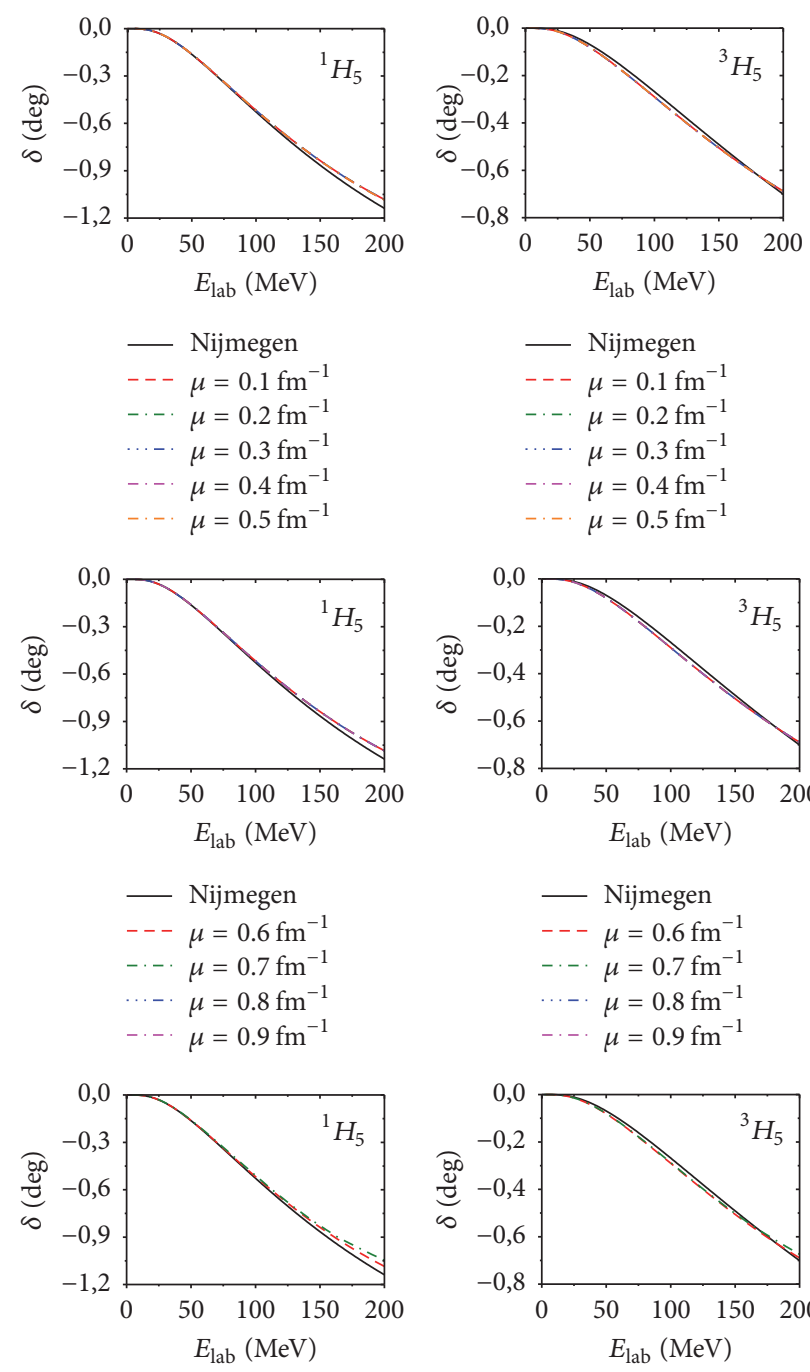

$$
\begin{aligned}
& \text { - Nijmegen } \\
& ---\mu=1.0 \mathrm{fm}^{-1} \\
& -\cdots \quad \mu=2.0 \mathrm{fm}^{-1}
\end{aligned}
$$

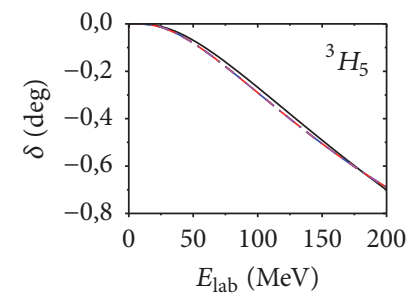

$$
\begin{aligned}
& \text { _ Nijmegen } \\
& \text { - - } \mu=0.6 \mathrm{fm}^{-1} \\
& \text {-.. } \mu=0.7 \mathrm{fm}^{-1} \\
& \text { … } \mu=0.8 \mathrm{fm}^{-1} \\
& \text {-.. } \mu=0.9 \mathrm{fm}^{-1}
\end{aligned}
$$

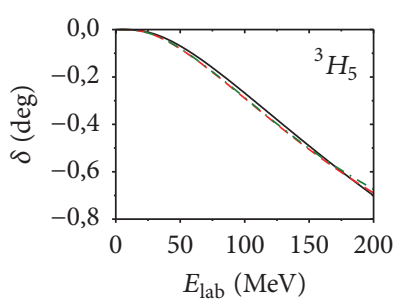

$$
\begin{aligned}
& \text { _ Nijmegen } \\
& ---\mu=1.0 \mathrm{fm}^{-1} \\
& -\cdot-\mu=2.0 \mathrm{fm}^{-1}
\end{aligned}
$$

Figure 9: (Color on-line) phase shifts in ${ }^{1} \mathrm{H}_{5}$ and ${ }^{3} \mathrm{H}_{5}$ channels calculated from the solution of the subtracted LS equation for the $K$-matrix with five subtractions for the N3LO-EGM potential for several values of the renormalization scale compared to the Nijmegen partial wave analysis.

only parameter of the EGM potential we changed was the cutoff $\Lambda \rightarrow \infty$. The EGM interaction depends on the SFR cutoff and the results are different if the SFR cutoff is modified, but this dependence is not related to the subtractions. We also believe that it is the SFR that drives the difference between the EM and EGM potentials as far as the renormalization scale dependence is concerned. Nevertheless, here we treated the SFR cutoff as an internal parameter of the EGM potential and looked only at the dependence of the phases on $\mu$.

The results for the N3LO-EM potential are displayed in Figures 1-4 for the uncoupled channels and in Figures 5 and 6 for the coupled channels. For the N3LO-EGM potential, the results are displayed in Figures 7-10 for the uncoupled
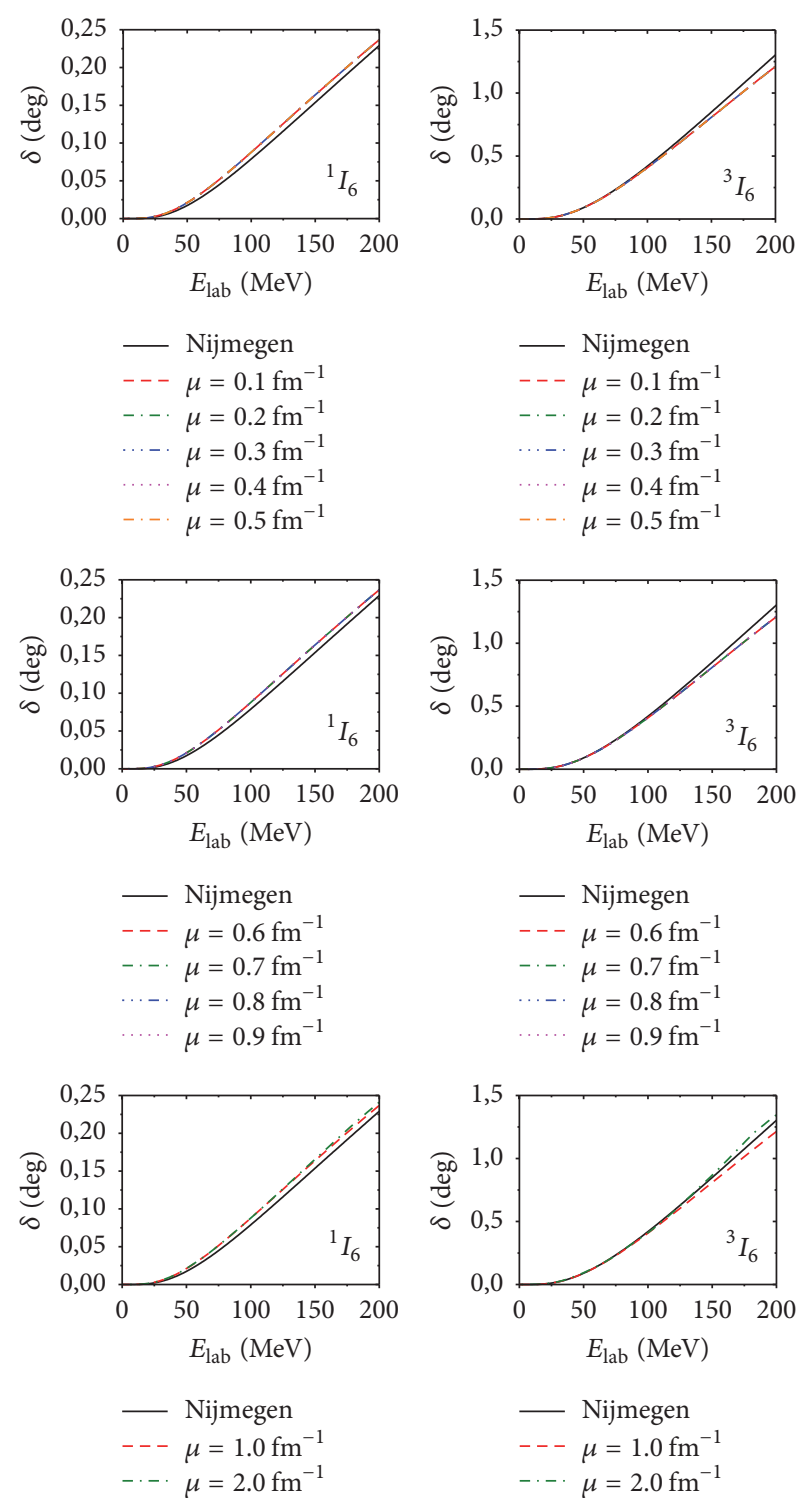

$$
\begin{aligned}
& \text { — Nijmegen } \\
& \text { - - } \mu=1.0 \mathrm{fm}^{-1} \\
& \text {-. . } \mu=2.0 \mathrm{fm}^{-1}
\end{aligned}
$$

FIGURE 10: (Color on-line) phase shifts in ${ }^{1} I_{6}$ and ${ }^{3} I_{6}$ channels calculated from the solution of the subtracted LS equation for the $K$-matrix with five subtractions for the N3LO-EGM potential for several values of the renormalization scale compared to the Nijmegen partial wave analysis.

channels and in Figures 11 and 12 for the coupled channels. The phase shifts are compared to the Nijmegen partial wave analysis [50]. For an updated high quality partial wave and error analysis, see the works from the Granada group [51-55].

Note that in the end we used different ranges of renormalization scales for EM and EGM and the reason is that the results for EGM converges faster as far as $\mu$ is concerned and there is no need to go above $2 \mathrm{fm}^{-1}$. In the case of EM, since there are still some variations in few waves for $\mu>2 \mathrm{fm}^{-1}$ we extended the range of the subtraction point to $10 \mathrm{fm}^{-1}$. We believe this is related to the differences between EM and EGM that makes EGM softer than EM. 


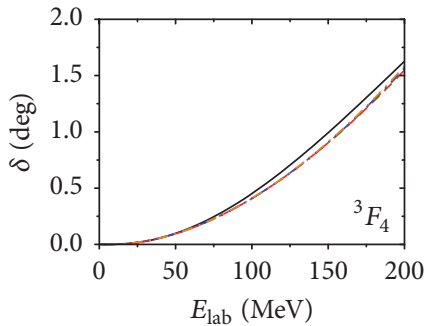

$$
\begin{aligned}
& \text { - Nijmegen } \\
& -\cdots \mu=0.1 \mathrm{fm}^{-1} \\
& \cdots-\mu=0.2 \mathrm{fm}^{-1} \\
& \cdots-\mu=0.3 \mathrm{fm}^{-1} \\
& \cdots \cdots=0.4 \mathrm{fm}^{-1} \\
& \cdots-\cdot \mu=0.5 \mathrm{fm}^{-1}
\end{aligned}
$$

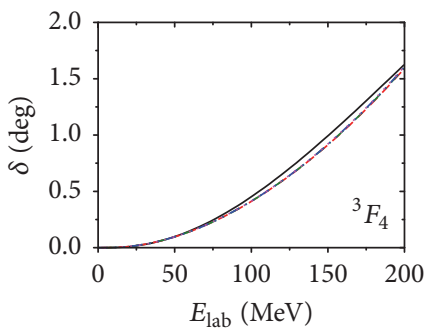

$$
\begin{array}{ll}
\text { — Nijmegen } \\
-\cdots=0.6 \mathrm{fm}^{-1} \\
\cdots \cdots=0.7 \mathrm{fm}^{-1} \\
\cdots-\mu=0.8 \mathrm{fm}^{-1} \\
\cdots \cdots=0.9 \mathrm{fm}^{-1}
\end{array}
$$

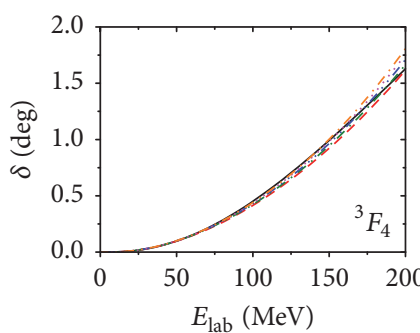

$$
\begin{aligned}
& \text { Nijmegen } \\
& -\cdots \mu=1.0 \mathrm{fm}^{-1} \\
& \cdots-\cdots=2.0 \mathrm{fm}^{-1} \\
& \cdots-\mu=3.0 \mathrm{fm}^{-1} \\
& \cdots \cdots=4.0 \mathrm{fm}^{-1} \\
& \cdots \mu
\end{aligned}
$$

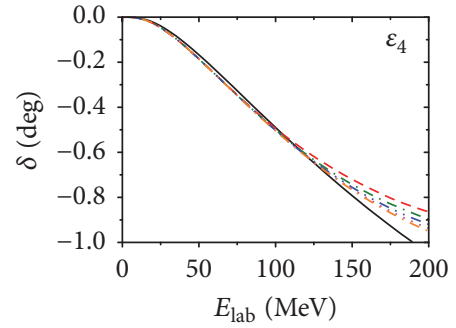

$$
\begin{aligned}
& \text { Nijmegen } \\
& -\cdots=0.1 \mathrm{fm}^{-1} \\
& \cdots \cdots=0.2 \mathrm{fm}^{-1} \\
& \cdots \cdots=0.3 \mathrm{fm}^{-1} \\
& \cdots \cdots=0.4 \mathrm{fm}^{-1} \\
& \cdots \mu=0.5 \mathrm{fm}^{-1}
\end{aligned}
$$

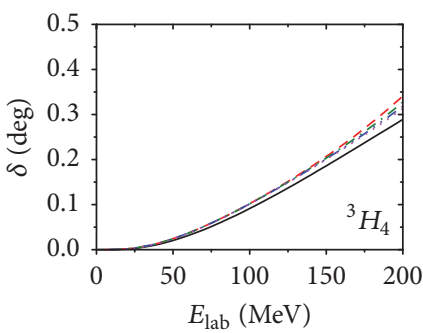

$$
\begin{aligned}
& \text { - Nijmegen } \\
& \text { - - } \mu=0.6 \mathrm{fm}^{-1} \\
& \text {-. } \mu=0.7 \mathrm{fm}^{-1} \\
& \cdots-\mu=0.8 \mathrm{fm}^{-1} \\
& \mu=0.9 \mathrm{fm}^{-1}
\end{aligned}
$$

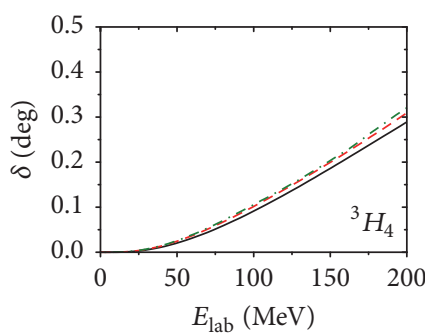

$$
\begin{aligned}
& \text { - Nijmegen } \\
& -\cdots \mu=1.0 \mathrm{fm}^{-1} \\
& -\cdots \mu=2.0 \mathrm{fm}^{-1}
\end{aligned}
$$

$$
\begin{aligned}
& \text { - Nijmegen } \\
& \text { - - } \mu=0.1 \mathrm{fm}^{-1} \\
& \text {-. } \mu=0.2 \mathrm{fm}^{-1} \\
& \cdots-\mu=0.3 \mathrm{fm}^{-1} \\
& \text { … } \mu=0.4 \mathrm{fm}^{-1} \\
& \text {-.. } \mu=0.5 \mathrm{fm}^{-1}
\end{aligned}
$$

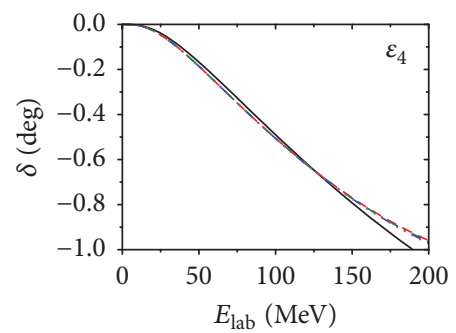

- Nijmegen

$--\mu=0.6 \mathrm{fm}^{-1}$

-.. $\mu=0.7 \mathrm{fm}^{-1}$

$\cdots \cdot \mu=0.8 \mathrm{fm}^{-1}$ $\mu=0.9 \mathrm{fm}^{-1}$

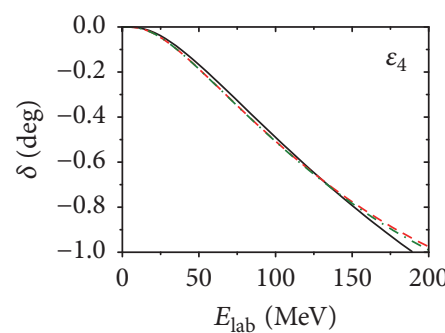

- Nijmegen

- - Scale 1.0

-. - Scale 2.0

FIGURE 11: (Color on-line) phase shifts in ${ }^{3} \mathrm{~F}_{4}-{ }^{3} \mathrm{H}_{4}$ coupled channels calculated from the solution of the subtracted LS equation for the $K$ matrix with five subtractions for the N3LO-EGM potential for several values of the renormalization scale compared to the Nijmegen partial wave analysis.

With few exceptions, in most of the channels we observe very small variation of the phases as we change the subtraction point, indicating that peripheral waves are nearly renormalization group invariant or fixed-points of the subtractive renormalization group. The exceptions are ${ }^{3} \mathrm{H}_{4}$ and ${ }^{3} I_{5}$ waves, which show slower convergence and ${ }^{3} G_{5}$ channel where the agreement with the partial wave analysis is only up to $\sim 50 \mathrm{MeV}$. Also, in the case of the EM potential, the phases for the ${ }^{3} F_{3}$ wave present some oscillations when the renormalization scale approaches $\mu \sim 10 \mathrm{fm}^{-1}$.

In this case of the coupled channels, the renormalization scheme is not failing but just requiring a slightly larger $\mu$ in the coupled channels, where we have the very singular tensor force. And this is what is different in the these channels compared to other $F$ waves. The case of the uncoupled triplet $3 F 3$ is somewhat different; the oscillation observed when 

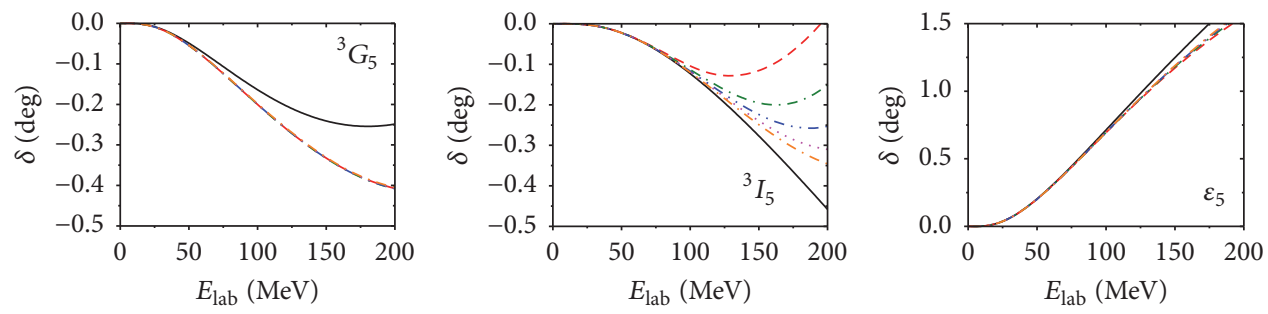
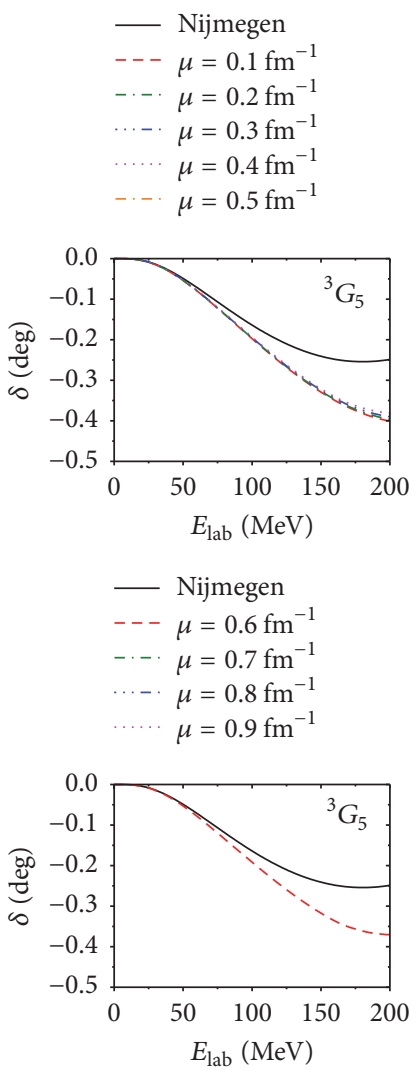

- Nijmegen
$---\mu=1.0 \mathrm{fm}^{-1}$
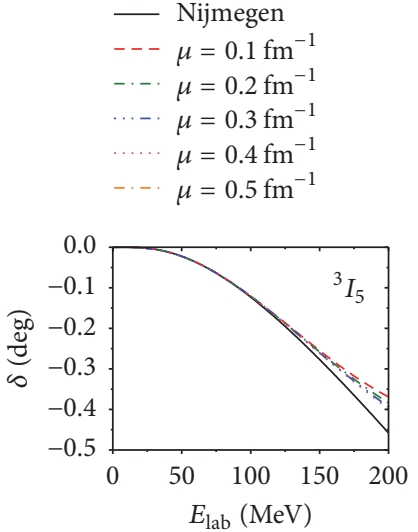

- Nijmegen

- - $\mu=0.6 \mathrm{fm}^{-1}$

-.. $\mu=0.7 \mathrm{fm}^{-1}$

... $\mu=0.8 \mathrm{fm}^{-1}$

$\mu=0.9 \mathrm{fm}^{-1}$

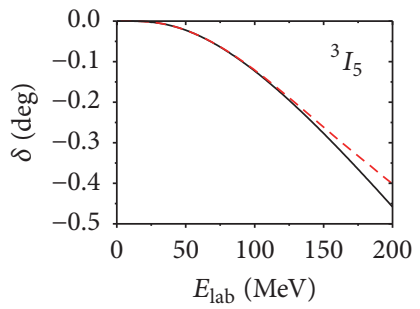

— Nijmegen

$$
\begin{array}{ll}
\text { —- Nijmegen } \\
---\mu=0.1 \mathrm{fm}^{-1} \\
\text { - - } \mu=0.2 \mathrm{fm}^{-1} \\
\cdots-\mu=0.3 \mathrm{fm}^{-1} \\
\cdots \cdots & \mu=0.4 \mathrm{fm}^{-1} \\
\cdots--\mu & \mu=0.5 \mathrm{fm}^{-1}
\end{array}
$$
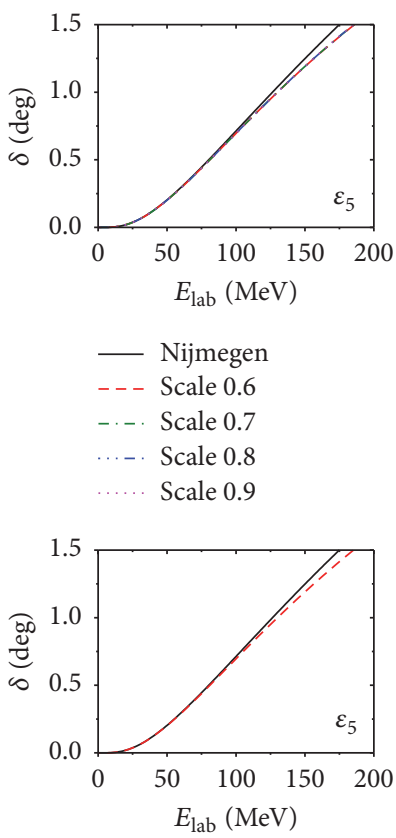

- Nijmegen
$---\mu=1.0 \mathrm{fm}^{-1}$

FIGURE 12: (Color on-line) phase shifts in ${ }^{3} G_{5}-{ }^{3} I_{5}$ coupled channels calculated from the solution of the subtracted LS equation for the $K$ matrix with five subtractions for the N3LO-EGM potential for several values of the renormalization scale compared to the Nijmegen partial wave analysis.

going from $\mu=6$ to $9 \mathrm{fm}^{-1}$ is related to the TPE without SFR of the EM potential. This oscillation is not seen in the $3 F 3$ wave when the EGM potential is used. An additional subtraction does not modify the results and the $\mu$ dependence in the these channels, so only the minimum number of subtractions needs to be performed (five at N3LO).

It is important to mention that the $\mu$-dependence of the phase shifts is encoded in the recursive driving terms $V_{\mu}^{(n)}$ since they all depend on the renormalization scale. In the case of cutoff regularization in configuration space [56], the cutoff radius $R_{S}$ dependence has been traced to the most singular part of the interaction which can be attraction or repulsion, depending on the channel. Here we observe more dependence on the coupled channels due to the very singular tensor force and the $F$-wave issue is due to the differences between the TPE components of the EM and EGM potentials.

\section{Conclusions and Outlook}

So far we have renormalized the N3LO interactions with multiple subtractions in peripheral channels considering an infinite cutoff $\Lambda=30 \mathrm{fm}^{-1}$. Only pions contribute to the nuclear force in these waves and the results are parameter-free.

The five subtractions performed in the kernel of the LS equation provide finite $K$-matrix in peripheral waves and the resulting phase shifts are rather independent of the subtraction point with the exception of ${ }^{3} \mathrm{H}_{4}$ and ${ }^{3} I_{5}$ waves 
where the fixed point is reached at approximately $\mu \sim 1 \mathrm{fm}^{-1}$ after the slow convergence shown in Figures 5, 6, 11, and 12.

The oscillation in the ${ }^{3} F_{3}$ channel suggests a closer look at this wave with the renormalization group flow equation that governs the driving terms (interactions) as the subtraction point is changed with the constraint of an invariant scattering amplitude.

The advances given by our approach when compared to cutoff regularization are as follows: the $N N$ force does not have to be modified prior to its insertion in the scattering equation; only the scattering equation is modified; the method is renormalization group invariant by construction and provides a nonrelativistic flow equation for the driving terms that tell us how they change when the renormalization scale is modified in order to keep the amplitude invariant. Finally, we would like to point out that the cutoff loses any physical significance since our results were obtained with an extremely large value $\Lambda=30 \mathrm{fm}^{-1}$.

\section{Competing Interests}

The authors declare that they have no competing interests.

\section{Acknowledgments}

The authors would like to thank financial support from FAPESP (Grant 2016/07061-3), CNPQ (Grant 306195/2015-1), and FAEPEX (Grant 3284/16).

\section{References}

[1] S. Weinberg, "Nuclear forces from chiral lagrangians," Physics Letters B, vol. 251, no. 2, pp. 288-292, 1990.

[2] S. Weinberg, "Effective chiral lagrangians for nucleon-pion interactions and nuclear forces," Nuclear Physics B, vol. 363, no. 1, pp. 3-18, 1991.

[3] S. Weinberg, "Three-body interactions among nucleons and pions," Physics Letters B, vol. 295, no. 1-2, pp. 114-121, 1992.

[4] C. Ordóñez, L. Ray, and U. van Kolck, "Nucleon-nucleon potential from an effective chiral Lagrangian," Physical Review Letters, vol. 72, no. 13, pp. 1982-1985, 1994.

[5] C. Ordóñez, L. Ray, and U. Van Kolck, "Two-nucleon potential from chiral Lagrangians," Physical Review C-Nuclear Physics, vol. 53, no. 5, article 2086, 1996.

[6] S. K. Adhikari, T. Frederico, and I. D. Goldman, "Perturbative renormalization in quantum few-body problems," Physical Review Letters, vol. 74, no. 4, pp. 487-491, 1995.

[7] S. K. Adhikari and T. Frederico, "Renormalization group in potential scattering," Physical Review Letters, vol. 74, no. 23, pp. 4572-4575, 1995.

[8] S. K. Adhikari and A. Ghosh, "Renormalization in nonrelativistic quantum mechanics," Journal of Physics A: Mathematical and General, vol. 30, no. 18, 1997.

[9] D. B. Kaplan, M. J. Savage, and M. B. Wise, "A new expansion for nucleon-nucleon interactions," Physics Letters B, vol. 424, no. 3-4, pp. 390-396, 1998.

[10] D. B. Kaplan, M. J. Savage, and M. B. Wise, "Two-nucleon systems from effective field theory," Nuclear Physics B, vol. 534, no. 1-2, pp. 329-355, 1998.
[11] D. B. Kaplan, M. J. Savage, and M. B. Wise, "Nucleon-nucleon scattering from effective field theory," Nuclear Physics B, vol. 478, pp. 629-659, 1996.

[12] P. F. Bedaque, H.-W. Hammer, and U. Van Kolck, "Renormalization of the three-body system with short-range interactions," Physical Review Letters, vol. 82, no. 3, pp. 463-467, 1999.

[13] B. Long and U. van Kolck, "Renormalization of singular potentials and power counting," Annals of Physics, vol. 323, no. 6, pp. 1304-1323, 2008.

[14] A. Nogga, R. G. E. Timmermans, and U. Van Kolck, "Renormalization of one-pion exchange and power counting," Physical Review C-Nuclear Physics, vol. 72, no. 5, Article ID 054006, 2005.

[15] M. C. Birse, J. A. McGovern, and K. G. Richardson, "A renormalisation-group treatment of two-body scattering," Physics Letters. B, vol. 464, no. 3-4, pp. 169-176, 1999.

[16] T. Barford and M. C. Birse, "Renormalization group approach to two-body scattering in the presence of long-range forces," Physical Review C, vol. 67, Article ID 064006, 2003.

[17] K. G. Richardson, M. C. Birse, and J. A. McGovern, "Renormalisation and power counting in effective field theories for nucleon-nucleon scattering," https://arxiv.org/abs/hep-ph/ 9708435.

[18] M. C. Birse, "Functional renormalization group for two-body scattering," Physical Review C, vol. 77, no. 4, 2008.

[19] M. Pavon Valderrama and E. Ruiz Arriola, "Renormalization of the $N N$ interaction with a chiral two-pion-exchange potential: central phases and the deuteron," Physical Review C, vol. 74, no. 5, Article ID 054001, 2006.

[20] M. Pavon Valderrama and E. Ruiz Arriola, "Renormalization of the NN interaction with a chiral two-pion exchange potential. II. Noncentral phases," Physical Review C, vol. 74, Article ID 064004, 2006.

[21] M. P. Valderrama and E. R. Arriola, "Renormalization of the deuteron with one pion exchange," Physical Review C, vol. 72, no. 5, 2005.

[22] M. P. Valderrama and E. R. Arriola, "Renormalization of $N N$ scattering with one pion exchange and energy dependent boundary conditions," Physical Review C, vol. 70, no. 4, Article ID 044006, 2004.

[23] D. R. Entem, E. R. Arriola, M. P. Valderrama, and R. MacHleidt, "Renormalization of chiral two-pion exchange NN interactions: momentum space versus coordinate space," Physical Review CNuclear Physics, vol. 77, no. 4, Article ID 044006, 2008.

[24] M. P. Valderrama, "Perturbative renormalizability of chiral twopion exchange in nucleon-nucleon scattering: $P$ and $D$ waves," Physical Review C, vol. 84, Article ID 064002, 2011.

[25] E. Epelbaum, H. Krebs, and U.-G. Meißner, "Improved chiral nucleon-nucleon potential up to next-to-next-to-next-toleading order," The European Physical Journal A, vol. 51, article 53, 2015.

[26] T. Frederico, V. S. Timóteo, and L. Tomio, "Renormalization of the one-pion-exchange interaction," Nuclear Physics A, vol. 653, no. 2, pp. 209-221, 1999.

[27] T. Frederico, A. Delfino, and L. Tomio, "Renormalization group invariance of quantum mechanics," Physics Letters. B. Particle Physics, Nuclear Physics and Cosmology, vol. 481, no. 1, pp. 143$150,2000$.

[28] V. Timóteo, T. Frederico, A. Delfino, and L. Tomio, "Recursive renormalization of the singlet one-pion-exchange plus pointlike interactions," Physics Letters B, vol. 621, no. 1-2, pp. 109-118, 2005. 
[29] V. Timóteo, T. Frederico, A. Delfino, and L. Tomio, "Subtractive renormalization of the next-to-leading order NN interaction," Nuclear Physics A, vol. 790, no. 1-4, pp. 406c-409c, 2007.

[30] V. S. Timóteo, T. Frederico, L. Tomio, and A. Delfino, "Renomalization of the nn interaction at nnlo: uncoupled peripheral waves," International Journal of Modern Physics E, vol. 16, no. 9, pp. 2822-2825, 2007.

[31] V. S. Timóteo, T. Frederico, A. Delfino, and L. Tomio, "Nucleonnucleon scattering within a multiple subtractive renormalization approach," Physical Review C, vol. 83, no. 6, Article ID 064005, 2011.

[32] S. Szpigel and V. S. Timóteo, "Power counting and renormalization group invariance in the subtracted kernel method for the two-nucleon system," Journal of Physics G: Nuclear and Particle Physics, vol. 39, no. 10, Article ID 105102, 2012.

[33] E. Epelbaum, W. Glöckle, and U.-G. Meißner, "Nuclear forces from chiral Lagrangians using the method of unitary transformation II: the two-nucleon system," Nuclear Physics A, vol. 671, no. 1-4, pp. 295-331, 2000.

[34] E. Epelbaum, W. Glöckle, and U.-G. Meißner, "Nuclear forces from chiral Lagrangians using the method of unitary transformation (I): formalism," Nuclear Physics A, vol. 637, no. 1, pp. 107134, 1998.

[35] E. Marji, A. Canul, Q. MacPherson et al., "Nonperturbative renormalization of the chiral nucleon-nucleon interaction up to next-to-next-to-leading order," Physical Review C, vol. 88, no. 5, Article ID 054002, 2013.

[36] D. R. Entem and R. Machleidt, "Accurate charge-dependent nucleon-nucleon potential at fourth order of chiral perturbation theory," Physical Review C, vol. 68, no. 4, Article ID 041001, 2003.

[37] R. Machleidt and D. R. Entem, "Chiral effective field theory and nuclear forces," Physics Reports, vol. 503, no. 1, pp. 1-75, 2011.

[38] E. Epelbaum, W. Glöckle, and U.-G. Meißner, "The twonucleon system at next-to-next-to-next-to-leading order," Nuclear Physics A, vol. 747, no. 2-4, pp. 362-424, 2005.

[39] E. Epelbaum, "Few-nucleon forces and systems in chiral effective field theory," Progress in Particle and Nuclear Physics, vol. 57, no. 2, pp. 654-741, 2006.

[40] E. Epelbaum, H.-W. Hammer, and U.-G. Meißner, "Modern theory of nuclear forces," Reviews of Modern Physics, vol. 81, no. 4, pp. 1773-1825, 2009.

[41] S. Bayegan, M. A. Shalchi, and M. R. Hadizadeh, "Three dimensional calculations of $N N$ bound and scattering states with a chiral potential up to $\mathrm{N}^{3}$ LO," Physical Review C, vol. 79, no. 5, Article ID 057001, 4 pages, 2009.

[42] S. Bayegan, M. Harzchi, and M. R. Hadizadeh, "Lowmomentum effective interaction in the three-dimensional approach," Nuclear Physics A, vol. 814, pp. 21-32, 2008.

[43] M. R. Hadizadeh and L. Tomio, "Three-dimensional lowmomentum interaction in two-body bound state calculations," Few-Body Systems, vol. 54, no. 12, pp. 2227-2232, 2013.

[44] E. F. Batista, S. Szpigel, and V. S. Timóteo, "Peripheral NN scattering from subtractive renormalization of chiral interactions," in Proceedings of the 36th Brazilian Workshop on Nuclear Physics, pp. 205-208, São Paulo, Brazil, September 2013.

[45] E. F. Batista, S. Szpigel, and V. S. Timóteo, "Renormalizing $\mathrm{N}_{3} \mathrm{LO}$ nucleon-nucleon interactions with multiple subtractions and infinite cutoff: the 3F4-3H4 and the 3G5 - 3I5 coupled channels," Few-Body Systems, vol. 55, no. 8-10, pp. 1049-1050, 2014.
[46] E. F. Batista, S. Szpigel, and V. S. Timóteo, "Running of the contact interactions in chiral N3LO potentials from subtractive renormalization," Journal of Physics: Conference Series, vol. 630, Article ID 012056, 2015.

[47] C. Yang, C. Elster, and D. R. Phillips, "Subtractive renormalization of the NN scattering amplitude at leading order in chiral effective theory," Physical Review C, vol. 77, no. 1, Article ID 014002, 2008.

[48] C. -J. Yang, C. Elster, and D. R. Phillips, "ubtractive renormalization of the chiral potentials up to next-to-next-to-leading order in higher NN partial waves," Physical Review C, vol. 80, Article ID 034002, 2009.

[49] C. J. Yang, Ch. Elster, and D. R. Phillips, "Subtractive renormalization of the $N N$ interaction in chiral effective theory up to next-to-next-to-leading order: $S$ waves," Physical Review $C$, vol. 80, no. 4, Article ID 044002, 2009.

[50] V. G. J. Stoks, R. A. M. Klomp, C. P. F. Terheggen, and J. J. de Swart, "Construction of high-quality NN potential models," Physical Review C, vol. 49, no. 6, pp. 2950-2962, 1994.

[51] R. Navarro Perez, J. E. Amaro, and E. Ruiz Arriola, "Partialwave analysis of nucleon-nucleon scattering below the pionproduction threshold," Physical Review C, vol. 88, no. 2, Article ID 024002, 7 pages, 2013.

[52] R. Navarro Perez, J. E. Amaro, and E. Ruiz Arriola, "Erratum: Partial-wave analysis of nucleon-nucleon scattering below the pion-production threshold [Physical Review C, vol. 88, Article ID 024002, 2013]," Physical Review C, vol. 88, no. 6, Article ID 069902, 2013.

[53] R. Navarro Perez, J. E. Amaro, and E. Ruiz Arriola, "Coarsegrained potential analysis of neutron-proton and proton-proton scattering below the pion production threshold," Physical Review C, vol. 88, no. 6, Article ID 064002, 31 pages, 2013.

[54] R. Navarro Perez, J. E. Amaro, and E. Ruiz Arriola, "Erratum: coarse-grained potential analysis of neutron-proton and proton-proton scattering below the pion production threshold [Phys. Rev. C 88, 064002 (2013)]," Physical Review C, vol. 91, Article ID 029901, 2015.

[55] R. Navarro Pérez, J. E. Amaro, and E. R. Arriola, "Coarsegrained NN potential with chiral two-pion exchange," Physical Review C, vol. 89, Article ID 024004, 2014.

[56] M. Pavon Valderrama and E. Ruiz Arriola, "Renormalization of the NN interaction with a chiral two-pion exchange potential. II. Noncentral phases," Physical Review C, vol. 74, no. 6, Article ID 064004, 2006. 

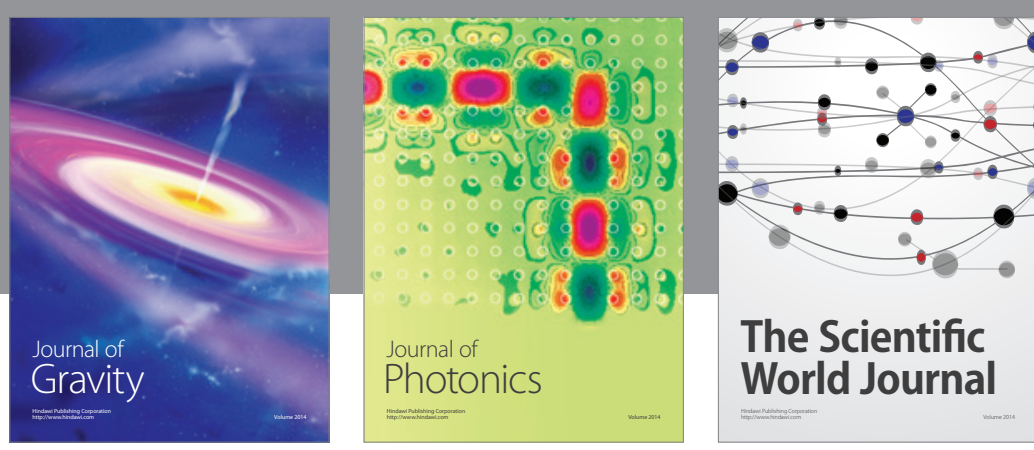

The Scientific World Journal
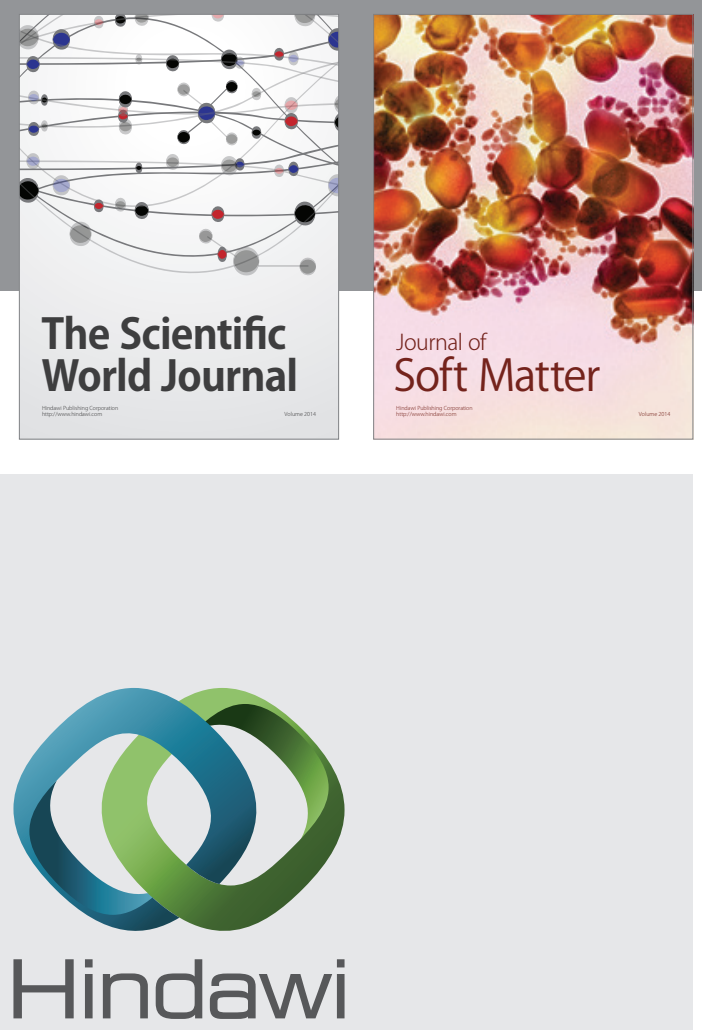

Submit your manuscripts at

https://www.hindawi.com
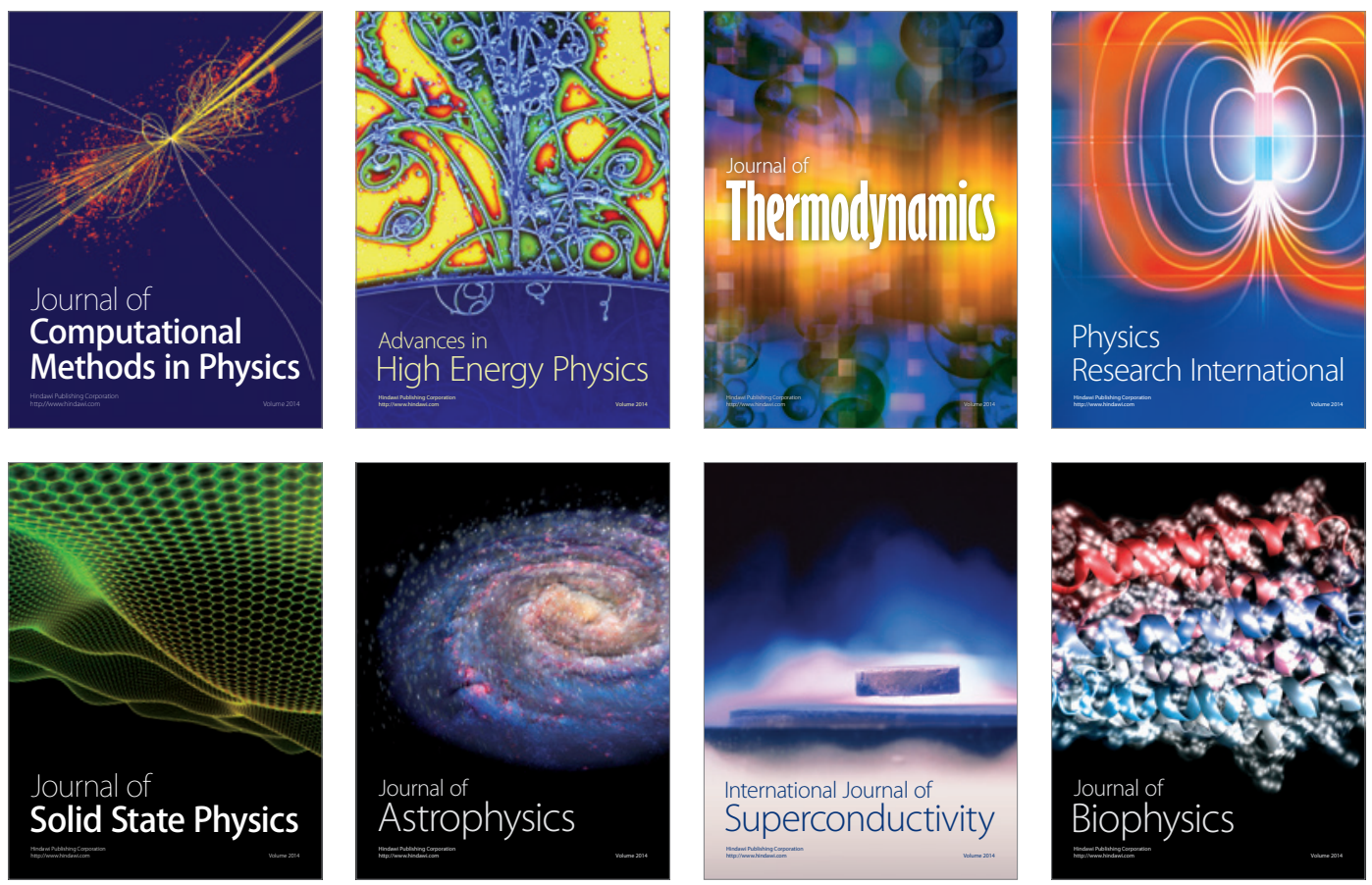
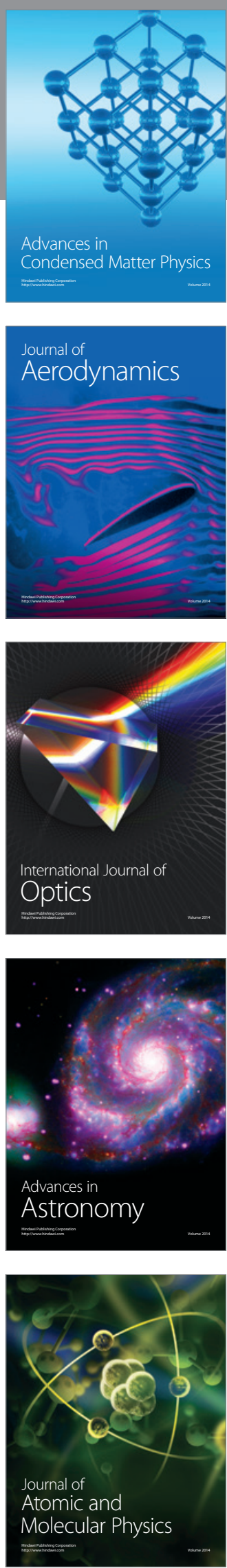